\title{
Koalitionsbildung in deutschen Großstädten: Empirische Befunde aus Nordrhein-Westfalen*
}

\author{
Kurzfassung
}

Studien zu lokaler Koalitionsbildung in westeuropäischen Staaten zeigen, dass sich Koalitionsbildungstheorien auch auf die lokale Ebene übertragen lassen. Umso erstaunlicher ist es, dass sowohl die „klassischen“ Theorien der Koalitionsbildung als auch institutionelle und kontextuelle Erklärungsfaktoren zur Koalitionsbildung in Mehrebenensystemen bisher nicht auf das kommunalpolitische System Deutschlands übertragen worden sind. Im vorliegenden Beitrag werde ich deshalb zunächst der Frage nachgehen, inwieweit sich die Koalitionsbildungsmuster auf der kommunalen, regionalen und nationalen Ebene in Deutschland unterscheiden. In einem zweiten Schritt werde ich dann die Erklärungsfaktoren kommunaler Koalitionsbildung bestimmen. Ich teste meine Erwartungen hinsichtlich der Muster kommunaler Koalitionsbildung in nordrhein-westfälischen Großstädten für den Zeitraum 1999-2009. Meine Ergebnisse zeigen, dass sowohl die Koalitionsbildungsmuster als auch die Determinanten kommunaler Koalitionsbildung denjenigen auf Bundesund Landesebene ähneln. Darüber hinaus spielt auch die Parteizugehörigkeit des direkt gewählten Oberbürgermeisters eine entscheidende Rolle bei der Bildung kommunaler Koalitionen.

* Ich danke den Teilnehmern der 3-Länder-Tagung Politikwissenschaft „Politik der Vielfalt“ (DVPW, ÖGPW, SVPW), die im September 2013 an der Universität Innsbruck stattfand, sowie insbesondere den beiden anonymen Gutachtern für hilfreiche Anmerkungen und Verbesserungsvorschläge. Besonderer Dank auch an Christian Rademacher für die Überlassung einzelner Daten zu den Kommunalwahlen in nordrhein-westfälischen Großstädten. 


\section{Inhalt}

1. Einleitung 110

2. Parteienwettbewerb und Koalitionsbildung auf der kommunalen Ebene 112

3. Theoretische Überlegungen und Hypothesen 115

a) „Klassische“ Theorien der Koalitionsbildung 115

b) Institutionelle und kontextuelle Faktoren der Koalitionsbildung $\quad 117$

4. Fallauswahl, Datengrundlage und methodische Vorgehensweise 120

5. Empirische Analysen 126

6. Fazit 133

\section{Einleitung}

Ende 2013 startete die Kommunalpolitische Vereinigung der CDU des Landes Nordrhein-Westfalen (KPV/NRW) den Wahlkampf der Christdemokraten für die nordrhein-westfälischen Kommunalwahlen am 25. Mai 2014 mit der Forderung, dass speziell in den zahlreichen Großstädten Koalitionen aus SPD, DIE LINKE und Bündnis 90/Die Grünen verhindert werden müssten. Vonseiten der Christdemokraten wird hierbei befürchtet, dass diese rot-rot-grünen Bündnisse eine Signalwirkung für zukünftige Bundes- und Landtagswahlen haben könnten. ${ }^{1}$ Inwieweit ist eine solche Befürchtung begründet? Einerseits werden kommunale Wahlen gerade in größeren Gebietseinheiten zunehmend medial begleitet. So werden Oberbürgermeister- oder auch Stadtratswahlen in Landeshauptstädten und anderen Großstädten immer mehr auch auf ihre landes- und bundespolitischen Auswirkungen hin beleuchtet. Andererseits wird konstatiert, dass sich insbesondere in den Großstädten „das politische System am deutlichsten dem der Länder und des Bundes angeglichen" habe und die dort gebildeten Koalitionen dabei weitgehend den Koalitionsmustern auf der Landesebene entsprechen würden (Zeuner/Wischermann 1995: 267). Dieser Befund wird allerdings zum einen mit Blick auf die nicht unerhebliche Anzahl an Koalitionen zwischen CDU und Bündnis 90/Die Grünen in den letzten zwanzig Jahren in mehreren Großstädten herausgefordert. ${ }^{2}$ Zum anderen zeigen neuere Studien, dass sich die kommunalen Parteiensysteme sowohl untereinander

1 http://www.kpv-nrw.de/3596-starke-cdu-starke-kommunen-kommunalwahlauftakt-der-nrw-cduund-52-delegiertenversammlung-der-kpv-nrw.html (Stand: 22.2.2014).

2 Lässt man Hamburg aufgrund seiner Sonderstellung als Stadtstaat außen vor, so bildeten sich seit 1994 in 12 Großstädten insgesamt 13 schwarz-grüne Koalitionen auf Basis einer Koalitionsvereinbarung. 
als auch im Vergleich mit dem Bundesparteiensystem und den Landesparteiensystemen voneinander unterscheiden (Schniewind 2010; Vetter/Kuhn 2013).

Während für andere westeuropäische Staaten bereits empirische Analysen lokaler Koalitionsbildungsprozesse existieren - und damit die Übertragbarkeit der Koalitionstheorien auf die lokale Ebene gezeigt werden konnte -, gibt es diesbezüglich für die deutsche Kommunalebene eine Forschungslücke. ${ }^{3}$ Diese Forschungslücke ist angesichts der Vorteile lokaler Koalitionsbildungsstudien erstaunlich, deren Durchführung immer wieder gefordert wird (siehe Buzogány/Kropp 2013; Laver/ Schofield 1998). Die Analyse kommunaler Koalitionen ist unter anderem dazu geeignet, für die nationale Ebene entwickelte Koalitionstheorien mit neuen Daten zu testen, bei gleichzeitiger Konstanthaltung institutioneller und kultureller Variablen (Giannetti/Benoit 2009: 4). Das Analyseinteresse wendet sich damit weg vom „methodologischen Nationalismus" und seiner Fokussierung auf den Nationalstaat (Jeffery 2008) und nimmt subnationale Einheiten vergleichend in den Blick (Snyder 2001). Dieser Beitrag soll ein erster Schritt in Richtung vergleichender Analysen kommunaler Koalitionsbildungsprozesse in deutschen Gemeinden und Städten sein.

Die Voraussetzungen zur Koalitionsbildung sind in den deutschen Kommunen gegeben. Mit dem Stadtrat existiert eine Arena für das Koalitionsspiel, die politischen Akteure können auch auf kommunaler Ebene reizvolle Posten besetzen, und kommunalpolitische Entscheidungen werden zunehmend von den politischen Akteuren in ihrem Sinne programmatisch und ideologisch beeinflusst (Brearey 1989: 285). Insbesondere die Großstädte mit mehr als 100.000 Einwohnern, die sich durch eine hohe „Parteipolitisierung“ (Wehling 1991) des politischen Wettbewerbs auszeichnen, sind dabei für die Analyse kommunaler Koalitionsbildung geeignet.

Bei der empirischen Analyse werden einerseits die ,klassischen“ Koalitionstheorien des office- und policy-seeking betrachtet, andererseits werden auch institutionelle und kontextuelle Faktoren in die Analyse miteinbezogen, die sich aus dem „quasi-präsidentiellen“ (Stoiber/Egner 2008) bzw. „semi-präsidentiellen“ (Bäck 2005) kommunalpolitischen System hinsichtlich der Direktwahl des Oberbürgermeisters sowie durch die Stellung der Kommunen im deutschen Mehrebenensystem ergeben. Dabei werden die Koalitionsbildungen in den 29 Großstädten in Nordrhein-Westfalen zwischen 1999 und 2009 analysiert, da sich insbesondere das kommunalpolitische System Nordrhein-Westfalens durch einen konkurrenzdemokrati-

3 So wurden lokale Koalitionsbildungsprozesse in Belgien (Geys/Heyndels/Vermeir 2006), Dänemark (Serritzlew/Skjæveland/Blom-Hansen 2008; Serritzlew/Blom-Hansen/Skjæveland 2010; Skjæveland/Serritzlew/Blom-Hansen 2007), Großbritannien (Laver/Rallings/Thrasher 1987, 1998), den Niederlanden (Denters 1985; Steunenberg 1992), Norwegen (Gravdahl 1998; Martinussen 2002), Portugal (Camões/Mendes 2009) und Schweden (Bäck 2003, 2008, 2009) analysiert. 
schen Parteienwettbewerb auszeichnet, der demjenigen auf Bundes- und Landesebene ähnelt (Holtkamp 2008). Die nordrhein-westfälischen Großstädte eignen sich damit am besten für einen ersten Test, inwieweit sich die Koalitionstheorien auf die kommunale Ebene in Deutschland übertragen lassen.

Die Ergebnisse zeigen, dass sich die Muster der Koalitionsbildung zwischen den einzelnen politischen Ebenen sowohl hinsichtlich der jeweiligen Koalitionskonstellationen als auch mit Blick auf die Determinanten der Koalitionsbildung ähneln. Es spielen einerseits office- und policy-basierte Faktoren eine Rolle, andererseits tragen auch (lokale) institutionelle Faktoren wie die Parteizugehörigkeit des direkt gewählten Oberbürgermeisters zur Erklärung der kommunalen Koalitionsbildung bei. Diese Ergebnisse verdeutlichen, dass die zur Erklärung nationaler Koalitionsbildungsprozesse entwickelten Koalitionstheorien auch auf der kommunalen Ebene in Deutschland Erklärungskraft besitzen.

Der Beitrag gliedert sich wie folgt: Im nächsten Abschnitt wird ein Überblick über den Parteienwettbewerb und die Koalitionsbildung auf der kommunalen Ebene in Deutschland gegeben. Anschließend werden die theoretischen Überlegungen und Hypothesen zur kommunalen Koalitionsbildung präsentiert. Im vierten Abschnitt wird einerseits die Fallauswahl näher begründet, andererseits werden die Datenbasis und die verwendeten Methoden vorgestellt. Im fünften Abschnitt werden die Ergebnisse der empirischen Analysen präsentiert und diskutiert. Abschließend werden die Ergebnisse des Beitrags zusammengefasst sowie ihre Verallgemeinerbarkeit für Koalitionsbildungsprozesse in anderen Kommunalsystemen und kleineren Gemeinden und Städten diskutiert.

\section{Parteienwettbewerb und Koalitionsbildung auf der kommunalen Ebene}

Im Unterschied zu den 1950er und 1960er Jahren werden Kommunalwahlen von den Parteien inzwischen als „Stimmungsbarometer“ und die einzelnen Gemeinden und Städte als zu gewinnende „Bastionen“ betrachtet (Kuschke/Cryns 1984: 60). Dies ist zum einen durch die „Parlamentarisierung der Kommunalpolitik“ (Frey/ Naßmacher 1975), in deren Zuge sich auch die kommunalen Vertretungskörperschaften in Richtung kommunaler „Parlamente“ entwickelten (Ott 1994; SchmidtEichstaedt 1985), zum anderen auch durch die deutliche Ausweitung der legislativen Kompetenzen kommunaler Vertretungskörperschaften seit den 1970er Jahren bedingt (Bogumil/Holtkamp 2006: 67).

Parteien haben auch auf der kommunalen Ebene eine herausgehobene Bedeutung bei der Willensbildung (Gabriel 2000: 15). Die Entscheidungsfindung der Gemeinde- und Stadträte bei der Auswahl von Handlungsoptionen wird dabei in der Regel 
auch von parteipolitischen Interessen und Überzeugungen geleitet (Holtmann 2002: 407). Darüber darf auch nicht die Tatsache hinwegtäuschen, dass Entscheidungen häufig einstimmig getroffen werden. Allen Entscheidungen gehen intensive und teils kontroverse Debatten innerhalb der Fraktionen und zwischen den einzelnen Ratsmitgliedern voraus, bei denen auch die Fraktionsdisziplin eine bedeutende Rolle spielt (Gehne/Holtkamp 2005; Tausendpfund 2013; van Deth/Tausendpfund 2013). Die Interessen und Überzeugungen der lokalen politischen Akteure sind dabei nicht losgelöst von den parteipolitischen Gegensätzen auf Bundes- und Landesebene zu sehen. Die klassische Links-Rechts-Einstufung der Parteien und ihrer Mitglieder findet auch auf der kommunalen Ebene ihre Entsprechung und hat Auswirkungen für die zu verfolgenden Interessen und den policy output (Debus/ Knill/Tosun 2012, 2013 a, 2013 b; Egner/Krapp/Heinelt 2013: 95; Goerres/Tepe 2013). Damit zeichnen sich auch auf der Kommunalebene die bereits auf Bundesund Landesebene erkennbaren Linien des Parteienwettbewerbs und der Parteiensysteme ab (Holtmann 2002: 418). ${ }^{4}$ Die kommunalen Parteiensysteme wurden dabei durch die Wahlerfolge der (nationalen) Parteien in den letzten Jahrzehnten zunehmend parteipolitisierter. Die „Parteipolitisierung“, das heißt die Höhe der Stimmengewinne der nationalen Parteien bei Kommunalwahlen, steigt unter anderem mit der Gemeindegröße an (Wehling 1991) und zeigt sich deshalb insbesondere in den Großstädten.

Je größer eine Kommune ist, desto mehr beeinflussen die Parteien das politische Geschehen, desto weniger werden Sachentscheidungen einstimmig getroffen und desto eher bestehen damit für die Akteure Anreize zur Koalitionsbildung. Gerade die Stadträte in den Großstädten können am ehesten als „Parlamente“ bezeichnet werden, in denen Koalitionen gebildet und Koalitionsvereinbarungen unterzeichnet werden, und somit eine Aufteilung des Stadtrats in Mehrheits- und Oppositionsfraktionen geschaffen wird (Egner/Krapp/Heinelt 2013; Gabriel 1984). Koalitionsspiele finden folglich im Stadtrat statt (Brearey 1989: 285).

Aufgrund der institutionellen Ausgestaltung der ,quasi-präsidentiellen“ (Stoiber/ Egner 2008) oder ,semi-präsidentiellen“ (Bäck 2005) kommunalpolitischen Systeme in den deutschen Bundesländern sind Stadtratskoalitionen jedoch nicht eins zu eins vergleichbar mit Koalitionen auf der Bundes- und Landesebene. Während das

4 Die auf der kommunalen Ebene stark vertretenen freien Wählergemeinschaften sind hierbei auch kein Grund, der Kommunalebene einen politischen Charakter abzusprechen. Diese sogenannten „Rathausparteien“"weisen zum einen ,,in Struktur und Handeln“ bereits seit längerem ähnliche Züge wie die etablierten Parteien auf (Holtmann 2004: 235), zum anderen beschränken sich zumindest die Freien Wähler nicht mehr auf die Repräsentation kommunalpolitischer Belange, sondern sind Mitglied des bayerischen Landtags und traten auch bei der Bundestagwahl 2013 an. 
Auseinanderbrechen einer Koalitionsregierung auf nationaler Ebene eine unmittelbare Neuformierung der Regierung nach sich ziehen muss, kann das Ende einer Koalition im Stadtrat zwei Folgen haben: Erstens können die kommunalpolitischen Akteure eine andere Stadtratskoalition bilden. Zweitens können sie aber auch darauf verzichten und sich für jede Entscheidung im Stadtrat unterschiedliche ad hoc-Koalitionen suchen.

Darüber hinaus handelt es sich bei Koalitionen auf Bundes- und Landesebene um exekutive Koalitionen, die sich auf eine Mehrheit im Parlament, das heißt auf eine legislative Koalition, stützen müssen (Laver 1989: 22). Auf kommunaler Ebene gibt es hingegen keine „Regierung“, die auf eine Mehrheit im Stadtrat angewiesen ist. Vor diesem Hintergrund wären kommunale Koalitionen deshalb eher als legislative Koalitionen anzusehen. Formal gesehen stellen zwar der Bürgermeister und die Verwaltung die Exekutive dar, während der Stadtrat hauptsächlich legislative Funktionen ausüben sollte - die tagtägliche Praxis zeigt jedoch, dass es eine Vielzahl von Interdependenzen zwischen der Verwaltung, dem Bürgermeister und dem Stadtrat gibt (Brearey 1989: 284; Hogwood 1999: 37), und somit eher eine duale Exekutive mit dem Bürgermeister (und der Verwaltung) auf der einen Seite und dem Stadtrat auf der anderen Seite existiert.

Warum sollten kommunalpolitische Akteure einen Anreiz haben, auf Dauer angelegte Bündnisse einzugehen und Koalitionsvereinbarungen zu unterschreiben? Die Argumente von Lupia und Strøm (2010: 61 f.) sowie Strøm und Nyblade (2007: 784 f.) zur Koalitionsbildung auf nationalstaatlicher Ebene lassen sich hierbei auch auf die kommunale Ebene übertragen: Erstens gehen kommunalpolitische Akteure Koalitionen ein und unterschreiben in den meisten Fällen auch eine Koalitions- oder Kooperationsvereinbarung (die die Erwartung einer dauerhaften Zusammenarbeit ausdrückt), um die Verhandlungs- und Transaktionskosten zwischen den Koalitionspartnern zu verringern. Im Unterschied zu ad hoc-Koalitionen reduziert sich der Zeitaufwand, von allen beteiligten Partnern akzeptierte Kompromisse zu finden. Zweitens ist die Unterzeichnung einer Koalitionsvereinbarung ein Signal an die Wähler, dass es keine abrupten Politikwechsel bei kommunalpolitischen Entscheidungen geben wird. Dies führt, drittens, zu einer größeren Glaubwürdigkeit kommunalpolitischer Entscheidungen und zu einer höheren Zurechenbarkeit kommunalpolitischer Verantwortung bei den Wählern. Kommunale Koalitionen, die auf Grundlage einer Koalitionsvereinbarung geschlossen werden, sind damit zwar nicht äquivalent zu Regierungskoalitionen auf Bundes- und Landesebene; sie sind aber formeller und verbindlicher ausgestaltet als reine legislative ad hoc-Koalitionen. 
Koalitionsbildung in deutschen Großstädten

\section{Theoretische Überlegungen und Hypothesen}

Meine theoretischen Erwartungen hinsichtlich der Bestimmungsfaktoren kommunaler Koalitionsbildung bauen auf den „klassischen“ Theorien der Koalitionsbildung auf und werden durch institutionelle und kontextuelle Faktoren erweitert, die sich aus der Stellung der Kommunen im deutschen Mehrebenensystem ergeben. In den folgenden Unterabschnitten werden die einzelnen Ansätze mit Blick auf die Koalitionsbildung in nordrhein-westfälischen Großstädten seit 1999 näher erläutert. Analysen lokaler Koalitionsbildung tragen dabei nicht nur zu einer erheblichen Ausweitung der Fallzahl bei (Laver/Rallings/Thrasher 1998: 352), sie ermöglichen gleichzeitig auch die Kontrolle zweier entscheidender Variablen: Der Faktor „Zeit“ und das institutionelle Arrangement werden konstant gehalten (Camões/Mendes 2009: 72; Laver/Rallings/Thrasher 1987: 503). Die Kontrolle des institutionellen Einflusses greift somit einen der wichtigsten Befunde der nationalen Koalitionsforschung auf, nämlich dass die Ergebnisse länderabhängig sind.

\section{a) „Klassische“ Theorien der Koalitionsbildung}

Die politischen Akteure verfolgen auch auf der kommunalen Ebene die Ziele des office-, policy- und vote-seeking: Sie wollen prestigeträchtige Ämter besetzen, ihre politischen Vorstellungen durchsetzen und eine größtmögliche Anzahl an Wählerstimmen gewinnen (Holtmann 1993; Skjæveland/Serritzlew/Blom-Hansen 2007: 727). Obwohl sich die verschiedenen Kommunalverfassungen von Bundesland zu Bundesland unterscheiden, von eher konsens- bis zu eher wettbewerbsorientierten Typen lokaler Demokratie (Freitag/Vatter 2010; Holtkamp 2008), bieten alle Kommunalverfassungen den Akteuren Anreize, miteinander in den Wettbewerb um politische Sachverhalte und kommunalpolitische Ämter zu treten. So besitzen die Kommunen weiterhin Selbstverwaltungsrechte, bei denen sie gestalterisch tätig werden können, und es ergeben sich trotz der zunehmenden rechtlichen Vorgaben höherer politischer Ebenen „bei der Ausführung der Aufgaben je nach Detaillierungsgrad der Rahmengesetze zum Teil beachtliche Handlungsspielräume“" (Bogumil/Holtkamp 2013: 18 f.). Die kommunalpolitischen Akteure verfügen damit über einen Gestaltungsspielraum hinsichtlich des policy output und es bieten sich somit Anreize zum policy-seeking. Insbesondere während der Wirtschafts- und Finanzkrise mussten viele kommunalpolitische Akteure in den Großstädten eine politische Abwägung zwischen verstärkter Haushaltskonsolidierung und der Aufrechterhaltung der sozialen Infrastruktur treffen. Aus Sicht der Ratsmitglieder spielt dabei auch die Durchsetzung des eigenen Parteiprogramms eine gewichtige Rolle (Egner/ Krapp/Heinelt 2013: 92 f.). 
Mit Blick auf die office-seeking-Aspekte kommunalpolitischer Akteure können als „funktionale Äquivalente“ zu den Ministerposten auf Bundes- und Landesebene die Dezernenten, hauptamtlichen Beigeordneten und stellvertretenden Bürgermeister angesehen werden, die beispielsweise in Nordrhein-Westfalen nicht vom Bürgermeister bestimmt, sondern direkt vom Stadtrat gewählt werden (Bogumil/Holtkamp 2013: 40; Egner/Krapp/Heinelt 2013: 86; Mellors/Brearey 1986: 288). Insbesondere in Nordrhein-Westfalen stellt auch das Amt als Ausschussvorsitzender „eine Prestigeposition dar, nach der man strebt, um Ansehen, Macht und Einfluss zu gewinnen“ (Wehling 2010: 358). Häufig halten die kommunalpolitischen Akteure in ihren Koalitionsvereinbarungen fest, wie einzelne Ämter zu besetzen sind. So verständigten sich Christdemokraten und Liberale 1999 in Bonn in ihrer Koalitionsvereinbarung darauf, dass der CDU zwei Bürgermeister zustehen sollten, die FDP dafür einen Ausschussvorsitz übernehmen und in kleineren Ausschüssen einen Sitz aus dem Kontingent der CDU bekommen sollte. ${ }^{5}$ In der Kooperationsvereinbarung für die „Ampel“-Koalition in Moers 2009 legten Bündnis 90/Die Grünen, FDP und SPD fest, welchen Parteien das jeweilige Vorschlagsrecht bei der Besetzung einzelner Beigeordneter und stellvertretender Bürgermeister zugesprochen wird. ${ }^{6}$ Zusätzlich kann für manche Parteien der Eintritt in eine kommunale Koalition wünschenswert sein, da, erstens, die Beteiligung an einer kommunalen Koalition dafür entschädigen kann, dass man auf den übergeordneten politischen Ebenen an keiner Regierung beteiligt ist, und, zweitens, insbesondere neue Parteien meistens zunächst auf der kommunalen Ebene in Erscheinung treten und dort ihren, first taste of power" genießen (Mellors/Brearey 1986: 288).

Die ,klassischen“ Koalitionstheorien basieren größtenteils auf den Annahmen des office- und policy-seeking. Den ämterorientierten Koalitionstheorien zufolge sollten potenzielle Koalitionen wahrscheinlicher sein, wenn sie minimal winning coalitions sind, das heißt wenn sie keine Parteien enthalten, die für die Parlamentsmehrheit nicht erforderlich sind, da ansonsten der Postenanteil der Koalitionsparteien sinken würde (von Neumann/Morgenstern 1944). Aus diesem Grund gibt es auch Anreize, eher minimum winning coalitions, das heißt kleinstmögliche Gewinnkoalitionen mit der geringsten Anzahl an Sitzen über der absoluten Mehrheit, oder Koalitionen mit der geringsten Anzahl an Parteien zu bilden (Leiserson 1968; Riker 1962). Darüber hinaus sollten solche Koalitionen eher gebildet werden, die die stärkste Partei enthalten, da diese eine starke Position bei Sondierungsgesprä-

5 Vergleiche Vereinbarung zwischen CDU und FDP-Fraktion für die Ratsperiode 1999 bis 2004.

6 Vergleiche Kooperationsvereinbarung zwischen den Fraktionen der SPD, der FDP und Bündnis 90/ Die Grünen im Rat der Stadt Moers für die Ratsperiode 2009-2014. 
chen und Koalitionsverhandlungen besitzt (Austen-Smith/Banks 1988; Bäck/Dumont 2008; Baron/Diermeier 2001; Baron/Ferejohn 1989). Alle office-seeking-Koalitionstheorien bauen damit auf dem Mehrheitskriterium auf, da in vielen Staaten eine Regierung nur ins Amt gelangen kann, wenn sie von einer absoluten Mehrheit im Parlament unterstützt wird (Strøm/Budge/Laver 1994). Auf der Kommunalebene bedarf es zwar keines investiture vote, dennoch ist anzunehmen, dass die kommunalpolitischen Akteure wie ihre bundes- und landespolitischen Pendants Minderheitskoalitionen aus Gründen von (vermuteter) Instabilität ablehnen. Demzufolge sollten potenzielle Koalitionen weniger wahrscheinlich sein, wenn sie über keine Mehrheit im Stadtrat verfügen. Im Unterschied zu den genannten Koalitionstheorien beziehen Theorien des policy-seeking zusätzlich (teilweise auch ausschließlich) die programmatisch-ideologischen Standpunkte der Parteien in die Analyse mit ein. Da diese auch auf der kommunalen Ebene eine entscheidende Rolle spielen können, sollte die Bildung einer potenziellen Koalition umso wahrscheinlicher sein, je geringer die programmatisch-ideologische Distanz zwischen den Koalitionspartnern ist (Axelrod 1970; De Swaan 1973; Grofman 1982).

Aufbauend auf diesen ,klassischen“ Koalitionstheorien lassen sich damit die folgenden sechs Hypothesen für die kommunale Koalitionsbildung formulieren:

H1: Potenzielle Koalitionen sollten wahrscheinlicher sein, wenn sie minimal winning coalitions sind.

H2: Potenzielle Koalitionen sollten wahrscheinlicher sein, wenn sie minimum winning coalitions sind.

H3: Potenzielle Koalitionen sollten wahrscheinlicher sein, wenn sie minimal winning coalitions mit der geringsten Anzahl an Parteien sind.

H4: Potenzielle Koalitionen sollten wahrscheinlicher sein, wenn sie die stärkste Partei enthalten.

H5: Potenzielle Koalitionen sollten weniger wahrscheinlich sein, wenn sie über keine Mehrheit im Stadtrat verfügen.

H6: Potenzielle Koalitionen sollten umso wahrscheinlicher sein, je geringer die ideologische Distanz zwischen den Koalitionspartnern ist.

\section{b) Institutionelle und kontextuelle Faktoren der Koalitionsbildung}

Die bis hierhin angeführten Koalitionstheorien vernachlässigen gewisse Beschränkungen (constraints) bei der Koalitionsbildung, die sich aus institutionellen und kontextuellen Faktoren ergeben und die die Anzahl der möglichen Koalitionskonstellationen reduzieren können. So beginnt das Koalitionsspiel nicht aus dem „Nichts“ - in den meisten Fällen gibt es eine bereits amtierende Koalition. Zahl- 
reiche Studien zeigen, dass diese Koalition gegenüber allen anderen Koalitionsmöglichkeiten im Durchschnitt eine höhere Wahrscheinlichkeit besitzt, als nächstes gebildet zu werden (Bäck/Dumont 2007; Franklin/Mackie 1983; Martin/Stevenson 2010; Strøm/Budge/Laver 1994). Dies sollte auch für die kommunale Ebene gelten.

Eine Analyse der kommunalen Koalitionsbildung in Deutschland muss darüber hinaus einen weiteren Akteur in den Blick nehmen, der als institutioneller constraint wirken kann: der direkt gewählte Oberbürgermeister, dessen Direktwahl durch den „Siegeszug der süddeutschen Kommunalverfassung“ (Knemeyer 1999) in den 1990er Jahren flächendeckend in den deutschen Kommunalsystemen eingeführt wurde. Direkt gewählte Oberbürgermeister äußern häufig ihre Präferenzen hinsichtlich der Koalitionsbildung im Stadtrat; so zum Beispiel die christdemokratischen Oberbürgermeister in Duisburg (2009) und Köln (2005), die sich deutlich gegen Koalitionen aus SPD, Bündnis 90/Die Grünen und DIE LINKE aussprachen. ${ }^{7}$ Mit anderen Worten: Die CDU-Oberbürgermeister waren gegen Koalitionen, die ihre eigene Partei nicht beinhalteten. Der SPD-Oberbürgermeister in Oberhausen (2009) äußerte hingegen, dass er einer großen Koalition mit der CDU und einer „rot-roten“ Koalition mit der Linkspartei ablehnend gegenüberstehen würde. ${ }^{8}$

Des Weiteren weist das Verhältnis zwischen direkt gewähltem Oberbürgermeister und dem Stadtrat im ,quasi-präsidentiellen“ (Stoiber/Egner 2008) bzw. „,semipräsidentiellen“ (Bäck 2005) System Parallelen zur Beziehung zwischen direkt gewählten Präsidenten und den Parlamenten in nationalen präsidentiellen und semipräsidentiellen Systemen auf. Kang (2009) zeigt für diese Systeme, dass die Partei des Präsidenten gegenüber anderen Parteien eine signifikant höhere Wahrscheinlichkeit besitzt, Teil der vom Parlament gebildeten Regierungskoalition zu werden. Kommunalpolitische Akteure könnten demnach einen Anreiz haben, die Partei des Oberbürgermeisters in die Stadtratskoalition mit aufzunehmen, um Verhandlungsund Transaktionskosten zu verringern, insbesondere vor dem Hintergrund, dass der Oberbürgermeister in Nordrhein-Westfalen automatisch auch Vorsitzender des Stadtrats ist. So beschneiden beispielsweise die Mehrheitsfraktionen in nordrheinwestfälischen Räten in „Kohabitationssituationen“ häufig die Kompetenzen des direkt gewählten (Ober-)Bürgermeisters, während dieser wiederum einzelne Ratsbeschlüsse verzögert oder überhaupt nicht umsetzt (Bogumil/Holtkamp 2013: 189; Gehne/Holtkamp 2005; Holtkamp 2008). Folge solcher „Kohabitationssituationen“ sind deutlich langsamere Entscheidungsprozesse bis hin zum politischen Stillstand.

\footnotetext{
7 Kölnische Rundschau (7.12.2005); www.derwesten.de/staedte/duisburg/adolf-sauerland-will-koalition-der-vernunft-id158797.html (Stand: 5.3.2013).

8 www.derwesten.de/staedte/oberhausen/es-riecht-sozialliberal-id163844.html (Stand: 5.3.2013).
} 
Somit sollten potenzielle Koalitionen weniger wahrscheinlich sein, wenn sie die Partei des Oberbürgermeisters nicht miteinbeziehen (Kohabitation).

Das Verhalten politischer Akteure in Mehrebenensystemen wird zusätzlich auch durch unterschiedliche Anreize und Einflüsse bestimmt, die sich aus dem Zusammenspiel der verschiedenen politischen Ebenen ergeben (Deschouwer 2003, 2006; Müller 2013; Swenden/Maddens 2009; van Houten 2009). Beispielsweise wird ausgehend vom Prinzipal-Agenten-Ansatz (Carey 2007, 2009; Müller 2000) hinsichtlich der Koalitionsbildung auf der regionalen Ebene argumentiert, dass regionale Parteigliederungen nach Wahlen vor dem Dilemma stehen, inwieweit sie Koalitionskonstellationen eingehen sollen, die zum jeweiligen Zeitpunkt nicht der Koalition auf der nationalen Ebene entsprechen. Koalitionen sind demnach wahrscheinlicher, wenn sie die Koalitionsmuster der übergeordneten politischen Ebene „kopieren“, um einerseits für die Akteure einen Verhandlungsvorteil aufgrund persönlicher Beziehungen und durch das Ausnutzen innerparteilicher Kanäle zu erzielen, um andererseits aber auch durch die Koalitionsentscheidungen auf den verschiedenen politischen Ebenen keine, ,gemischten Signale“ an die Wähler zu senden (Bäck u. a. 2013; Däubler/Debus 2009; Ştefuriuc 2009 a, 2009 b, 2013). Ein Blick auf die Diskussionen über eine mögliche Signalwirkung der ersten schwarz-grünen Koalition in einer deutschen Millionenstadt in Köln 2003 für zukünftige Koalitionen aus CDU und Bündnis 90/Die Grünen auf Landes- und Bundesebene (Kleinert 2004; Neu 2004) verdeutlicht, dass dieser Aspekt auch für die kommunale Ebene gelten könnte. Potenzielle Koalitionen sollten folglich wahrscheinlicher sein, wenn sie kongruent zu den Koalitionen auf Landesebene sind.

Diese Erwartung sollte jedoch von folgendem elektoralen constraint abhängig sein (Bäck 2003; Denters 1985; Downs 1998; Ştefuriuc 2009 a, 2009 b, 2013): der Ähnlichkeit zwischen kommunalem und regionalem Parteiensystem hinsichtlich der zur Wahl angetretenen Akteure sowie der Wahlergebnisse. Die Möglichkeiten der kommunalpolitischen Akteure, kongruente Koalitionen zur Landesregierung zu bilden, sollten von der Zusammensetzung der Stadträte im Vergleich zum Landesparlament abhängen: Ähnelt sich die Sitzverteilung bei der Kommunalwahl und bei der Landtagswahl, das heißt ist das kommunale Parteiensystem symmetrisch zum Landesparteiensystem, so ist die Wahrscheinlichkeit für die Bildung einer kongruenten Koalition höher, da die Koalitionskonstellationen eher dieselben sind (Ştefuriuc 2009 b: 186). Bildet Partei A mit Partei B die Landesregierung und haben beide Parteien bei der Stadtratswahl zusammen eine Mehrheit, dürfte es Partei A schwer fallen, die Wähler von einer Koalition mit Partei C zu überzeugen (vgl. Bäck u. a. 2013: 371 f.). Im Extremfall unterscheiden sich die Wahlergebnisse im kommunalen Parteiensystem so stark von den Landeswahlergebnissen, dass Partei A und/oder 
Partei B gar nicht im Stadtrat vertreten sind und die potenzielle Koalition zwischen beiden Parteien überhaupt nicht möglich ist. Potenzielle Koalitionen, die kongruent zur Landesregierung sind, sollten demnach umso unwahrscheinlicher sein, je asymmetrischer das jeweilige kommunale Parteiensystem ist.

Aufbauend auf diesen Überlegungen zu institutionellen und kontextuellen Faktoren bei der Koalitionsbildung lassen sich somit vier weitere Hypothesen formulieren:

H7: Potenzielle Koalitionen sollten wahrscheinlicher sein, wenn sie die amtierende Koalition sind.

H8: Potenzielle Koalitionen sollten weniger wahrscheinlich sein, wenn sie die Partei des Oberbürgermeisters nicht miteinbeziehen (Kohabitation).

H9: Potenzielle Koalitionen sollten wahrscheinlicher sein, wenn sie kongruent zur Landesregierung sind.

H10: Potenzielle Koalitionen, die kongruent zur Landesregierung sind, sollten in asymmetrischen Parteiensystemen unwahrscheinlicher sein.

\section{Fallauswahl, Datengrundlage und methodische Vorgehensweise}

Der vorliegende Beitrag analysiert die Koalitionsbildung in 29 nordrhein-westfälischen Großstädten für den Zeitraum 1999-2009. Als Großstädte werden alle Kommunen angesehen, die zum Stichtag 31.12.2010 eine Einwohnerzahl von mindestens 100.000 Einwohnern aufwiesen. Zur Identifizierung der Stadtratskoalitionen wurden sowohl die Wahlämter als auch die fünf großen Parteien (Bündnis 90/Die Grünen, CDU, DIE LINKE, FDP, SPD) in den jeweiligen Großstädten angeschrieben, mit der Bitte um Auskunft über etwaige Koalitionen im Stadtrat und mit der Bitte, schriftlich vorliegende Koalitionsvereinbarungen und die jeweiligen Kommunalwahlprogramme für den Zeitraum 1994 bis 2009 zu übermitteln. ${ }^{9}$ Diese Angaben wurden durch Onlinerecherchen hinsichtlich der Internetauftritte der Parteien ergänzt, um auch in denjenigen Fällen die Koalitionsvereinbarungen zu finden, in denen die Parteien nicht antworteten. Als Koalitionen werden zum einen alle Bündnisse gezählt, in denen eine schriftliche Kooperationsvereinbarung physisch vorliegt. Zum anderen werden auch solche Bündnisse als Koalitionen gezählt, in denen die Wahlämter und/oder die Parteien bestätigten, dass der Koalition eine schriftliche

9 Die Kommunalwahlen 1994 sind wegen der unterschiedlichen institutionellen Rahmenbedingungen (Geltung der Fünfprozenthürde; noch keine flächendeckende Einführung von Direktwahlen der Oberbürgermeister) nicht Bestandteil der folgenden Analysen. 
Vereinbarung zugrunde lag. ${ }^{10}$ Hierbei werden ausschließlich Koalitionen im Anschluss an Kommunalwahlen betrachtet. Koalitionen, die sich während der Legislaturperiode bildeten, beispielsweise nach dem Auseinanderbrechen einer anderen Koalition, werden nicht analysiert, da die Ausgangslage und die Präferenzen der kommunalpolitischen Akteure nicht vergleichbar mit den Situationen im direkten Anschluss an Kommunalwahlen sind.

Die Großstädte in Nordrhein-Westfalen bieten sich für einen ersten Test der Übertragbarkeit (nationaler) Koalitionstheorien auf die kommunale Ebene in Deutschland als ideale Fälle an. Erstens können durch die Analyse der kommunalen Koalitionsbildung in einem einzigen Bundesland die institutionellen Begebenheiten und der politisch-kulturelle Kontext konstant gehalten werden (Giannetti/Benoit 2009). Zweitens ist das kommunalpolitische System in Nordrhein-Westfalen ein Musterbeispiel für ein auf Wettbewerb ausgerichtetes politisches System, dem ein hoher Grad an Parteipolitisierung attestiert wird und dessen kommunales Parteiensystem am ehesten denjenigen auf Bundes- und Landesebene ähnelt (Gehne/Holtkamp 2005; Holtkamp 2008; Vetter/Kuhn 2013). Die Parteienkonkurrenz zwischen den kommunalpolitischen Akteuren ist insbesondere in Nordrhein-Westfalen sehr ausgeprägt (Bogumil 2001: 101). Die hohe Parteipolitisierung führt deshalb zu einer klaren Trennung in Mehrheits- und Oppositionsgruppen im Stadtrat, was wiederum ursächlich für ein einheitliches Abstimmungsverhalten der einzelnen Fraktionen ist. Leisten die Koalitionstheorien hier keinen (oder nur einen geringen) Beitrag zur Erklärung der Koalitionsbildungen, so ist es unwahrscheinlich, dass sie in weniger kompetitiven Kommunalsystemen eine größere Erklärungskraft besitzen könnten.

Drittens sind Parteipolitisierung und Koalitionsbildung eng mit der Gemeindegröße verbunden (Brearey 1989; Fried 1976). Je größer eine Kommune ist, desto mehr beeinflussen die Parteien das politische Geschehen und desto weniger werden Sachentscheidungen in der kommunalen Vertretungskörperschaft einstimmig getroffen. Damit steigen die Anreize zur Koalitionsbildung. Ein Drittel der deutschen Großstädte liegen in Nordrhein-Westfalen. Deshalb ist Nordrhein-Westfalen dasjenige Bundesland, das für eine Analyse kommunaler Koalitionsbildung am besten geeignet ist. Viertens bietet diese hohe Anzahl an Großstädten Möglichkeiten zur quantitativen Überprüfung einzelner Koalitionstheorien, die zum einen mit Blick auf nationale Koalitionsbildungsprozesse entwickelt, zum anderen aber auch hauptsächlich anhand nationaler Daten getestet wurden. Die hohe Fallzahl kommunaler

10 Dies umfasst nur wenige Fälle, in denen sich die Koalitionsparteien weigerten, die Kooperationsvereinbarung zu übermitteln, oder in denen die Vereinbarungen den Koalitionsparteien nicht mehr vorlagen. 
Koalitionen hilft dabei, dieses ,inzestuöse Verhältnis“ zwischen Theorieentwicklung und -überprüfung zu umgehen (Laver 1989: 16) und das große Potenzial zu nutzen, das den Analysen kommunaler Koalitionsbildungsprozesse zugeschrieben wird (Buzogány/Kropp 2013: 288; Laver/Schofield 1998: 9).

Fünftens wurde die Direktwahl des Oberbürgermeisters in Nordrhein-Westfalen erst 1999 eingeführt. Die „Aufwertung“ der Rolle des Oberbürgermeisters ist somit noch relativ neu und das Verhältnis zwischen Stadtratsmehrheit und den direkt gewählten Oberbürgermeistern fußt noch nicht auf jahrzehntelangen Pfadabhängigkeiten. So zeigt sich gerade in Nordrhein-Westfalen ein Spannungsverhältnis zwischen der ,exekutiven Vorherrschaft" der Oberbürgermeister und der weiterhin im Stadtrat vorherrschenden „Parteiherrschaft“ (Bogumil 2001: 245 f.), das auch durch die weitreichenden Rechte des Stadtrats gegenüber dem Oberbürgermeister bedingt wird (Bogumil/Holtkamp 2013: 33; Bogumil/Grohs/Holtkamp 2010: 801). Gerade in Nordrhein-Westfalen zeigen sich deshalb viele Probleme, die sich aus „Kohabitationssituationen", das heißt gegenläufigen Parteiorientierungen von Oberbürgermeister und Stadtratskoalition, ergeben können (Bogumil 2010; Florack 2010; Nienaber 2004).

Es wurde ein neuer Datensatz erstellt, der Informationen zu den Wahlergebnissen, zur Sitzverteilung im Stadtrat, zur jeweiligen Koalitionsbildung in den Großstädten und auf der Landesebene, zu den policy-Positionen der kommunalpolitischen Akteure anhand ihrer Wahlprogramme und zu den direkt gewählten Oberbürgermeistern enthält. Mit Blick auf die office-seeking-Faktoren und den vermuteten Amtsinhabervorteil werden jeweils Dummy-Variablen gebildet, die beispielsweise anzeigen, ob es sich bei potenziellen Koalitionen um minimal winning coalitions handelt oder nicht.

Die ideologische Heterogenität innerhalb potenzieller Koalitionen wird anhand der Veto-Spieler-Distanz gemessen (Tsebelis 1995, 2002). Hierfür werden die policy-Positionen der einzelnen kommunalpolitischen Akteure mithilfe des Wordscores-Verfahrens quantitativ-inhaltsanalytisch geschätzt (ausführlicher Laver/Benoit/Garry 2003; Lowe 2008). ${ }^{11}$ Die policy-Positionen der kommunalpolitischen Akteure werden auf einer wirtschaftspolitischen und auf einer gesellschaftspoliti-

11 Da sich bereits die policy-Positionen der Landesparteien von denjenigen der Bundesparteien deutlich unterscheiden (Bräuninger/Debus 2012; Müller 2013), ist eine Schätzung der kommunalpolitischen Positionen geeigneter als die Übernahme der nationalen Parteipositionen (vgl. Denters 1985; Laver/Rallings/Thrasher 1998; Steunenberg 1992). Surveybefragungen der Kommunalpolitiker zu den jeweiligen policy-Positionen der Parteien (Bäck 2003; Egner/Krapp/Heinelt 2013; Skjæveland/Serritzlew/Blom-Hansen 2007) sind für diesen großen Untersuchungszeitraum nicht möglich. 
schen Dimension bestimmt, da diese beiden Dimensionen den deutschen Parteienwettbewerb auf Bundes- und Landesebene maßgeblich strukturieren (Pappi 2009) und auch den kommunalen Parteienwettbewerb prägen sollten. Ob Steuergelder eher für eine neue Umgehungsstraße oder für eine bessere Ausstattung von Schulen ausgegeben werden, ist eine wirtschaftspolitische Entscheidung. Die hochgradig politisierten Diskussionen über den Bau von Moscheen sind ein Beispiel für die Bedeutung der gesellschaftspolitischen Dimension im kommunalen Parteienwettbewerb.

Für die Anwendung von Wordscores wird angenommen, dass politische Akteure Worte nicht zufällig, sondern in absichtsvoller Weise verwenden, um ,ideologische Signale“ (Pappi/Shikano 2004) an die eigene Wählerschaft zu senden. Zur Schätzung der policy-Positionen in den kommunalen Wahlprogrammen bedarf es gewisser „Referenztexte“, deren policy-Positionen, sog. „Referenzwerte“, bekannt sind und die strukturell den kommunalen Wahlprogrammen ähneln. Als „Referenztexte“ werden die Wahlprogramme von Bündnis 90/Die Grünen, CDU, FDP und SPD für die Landtagswahlen in Nordrhein-Westfalen 1990, 1995, 2000, 2005 und 2010 sowie das Landtagswahlprogramm der Partei DIE LINKE für die Landtagswahl 2010 verwendet. Die Zuordnung der jeweiligen „Referenzwerte“ zu den „Referenztexten" erfolgte mithilfe von Informationen zu den policy-Positionen nordrhein-westfälischer Landesparteien auf einer wirtschafts- und auf einer gesellschaftspolitischen Dimension (Bräuninger/Debus 2012). ${ }^{12}$

12 Für einzelne kommunale Parteien (Wählervereinigungen) mit fehlenden Kommunalwahlprogrammen wurden die policy-Positionen aus ihren kommunalen Grundsatzprogrammen geschätzt. In Fällen, in denen für eine Partei in einer Stadt nicht für jede Kommunalwahl ein Wahlprogramm vorliegt, wurde die policy-Position der vorherigen oder der nachfolgenden Wahl als Näherungswert genommen. In Fällen, in denen überhaupt kein Kommunalwahlprogramm einer Partei in einer Stadt vorliegt, wurde der Mittelwert aller policy-Positionen dieser Partei in den anderen analysierten Städten als Näherungswert imputiert. Für die PIRATEN, das ZENTRUM und die GRAUEN wurden die policy-Positionen aus dem Bundestagswahlprogramm 2002 (GRAUE), dem nordrheinwestfälischen Landtagswahlprogramm 2012 (PIRATEN) beziehungsweise dem nationalen Grundsatzprogramm (ZENTRUM) als Näherungswerte verwendet. Darüber hinaus wurden zur Bestimmung der policy-Positionen von „Antisystemparteien“ die auf Experteneinschätzungen beruhenden Resultate in Benoit und Laver (2006) verwendet und den kommunalen Parteiablegern zugeordnet, da Wordscores den ,extremistischen Charakter“ dieser Parteien nicht erfasst. Hierbei wurden DKP, DVU, NPD, Offensive D und REP wegen ihrer extremistischen ideologischen Orientierung als „Antisystemparteien“ identifiziert. Zusätzlich wurden auch PRO Köln und PRO NRW als rechtsextreme Bewegungen angesehen (Killguss/Peters/Häusler 2008; Peters/Sager/Häusler 2008). Da PRO Köln und PRO NRW nicht in den Daten von Benoit und Laver (2006) enthalten sind, wurde zur Bestimmung ihrer kommunalen Parteipositionen der Mittelwert der policy-Positionen von DVU, NPD und REP verwendet. Der Anteil gescorter Wörter in den Kommunalwahlprogrammen liegt durchschnittlich bei knapp 91 Prozent. 
Das Gesamtbild der Politikpositionen kommunalpolitischer Akteure weist zwar eine Ähnlichkeit mit den Positionen der Landes- und Bundesparteien auf, zumindest solange nur die Anordnung der kommunalen Parteien auf beiden Dimensionen betrachtet wird (vgl. Bräuninger/Debus 2012; Müller 2013; Pappi 2009). Die Analyse zeigt jedoch auch, dass es zum einen sowohl auf der wirtschafts- als auch auf der gesellschaftspolitischen Dimension große Unterschiede zwischen den Politikpositionen der Parteien gibt; zum anderen existieren auch innerhalb einzelner Parteien von Großstadt zu Großstadt Unterschiede bei den Politikpositionen (vgl. Abbildun-

Abbildung 1: Wirtschafts- und gesellschaftspolitische Positionen kommunalpolitischer Akteure in nordrhein-westfälischen Großstädten, 1999-2009

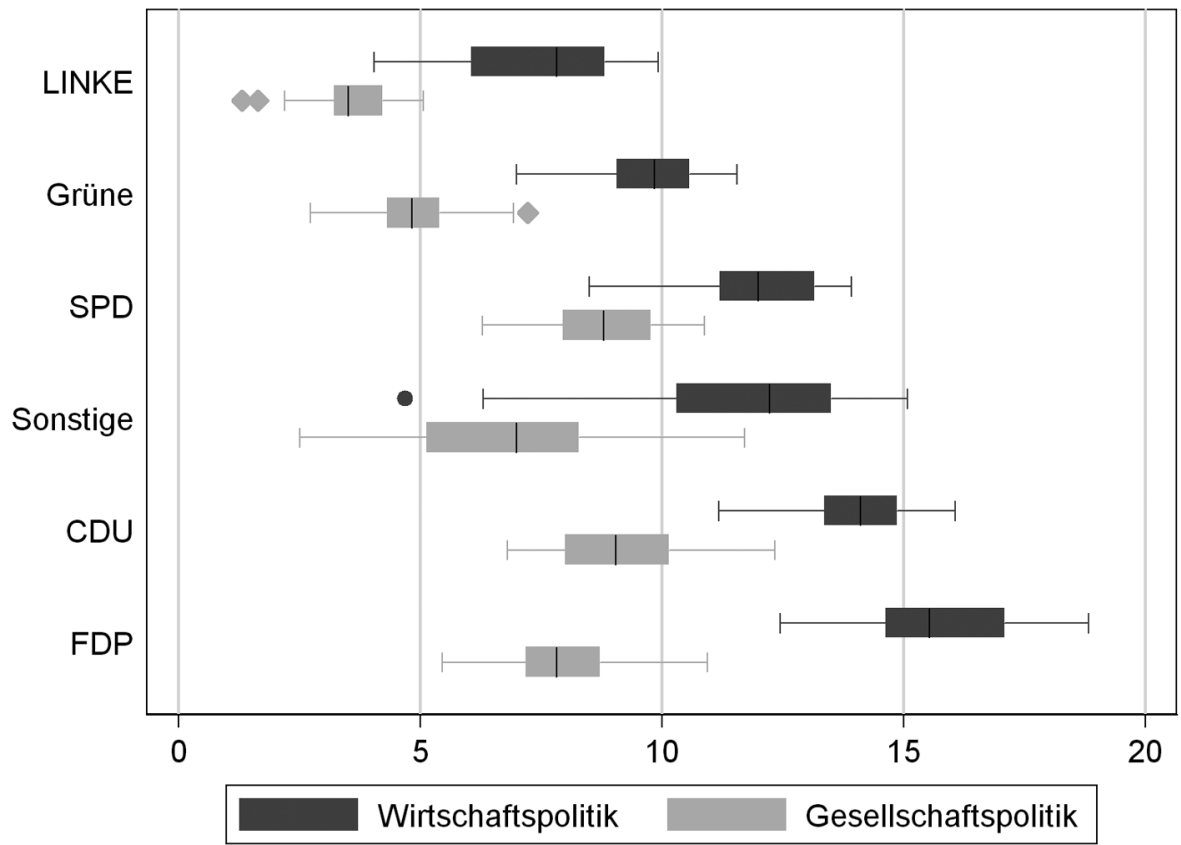

Anmerkung: Niedrige Werte zeigen eine wirtschaftspolitisch „linke“ und gesellschaftspolitisch ,progressive" Politikposition an, während hohe Werte wirtschaftspolitisch ,liberale“ und gesellschaftspolitisch „konservative“ Politikvorstellungen repräsentieren. 
gen 1 und 2). ${ }^{13}$ Die Ergebnisse sind ein Beleg dafür, dass es für die Analyse des großstädtischen Parteienwettbewerbs und der Koalitionsbildung angemessen ist, die kommunalen Politikpositionen der Parteien (und Wählervereinigungen) zu verwenden.

Abbildung 2: Wirtschafts- und gesellschaftspolitische Positionen der großen Parteien in nordrhein-westfälischen Großstädten, 1999-2009

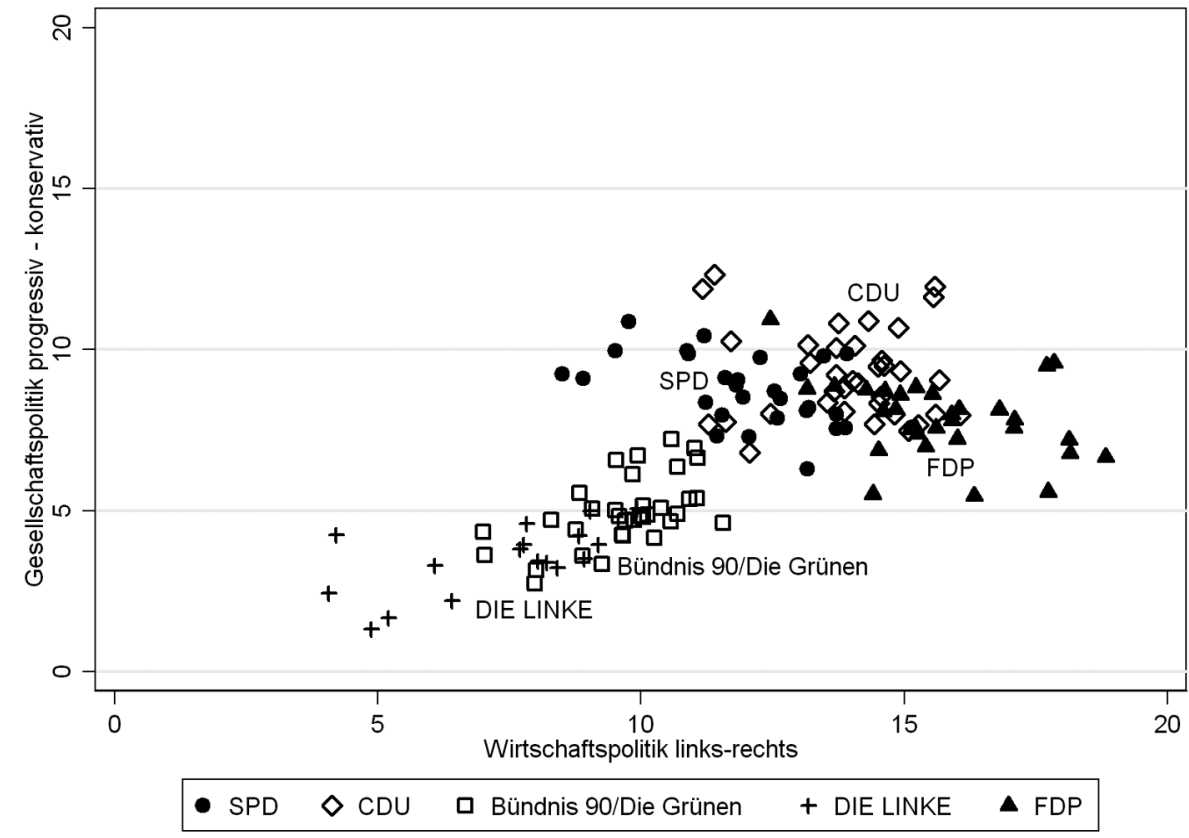

Anmerkung: Niedrige Werte zeigen eine wirtschaftspolitisch „linke“ und gesellschaftspolitisch „progressive“ Politikposition an, während hohe Werte wirtschaftspolitisch „liberale“ und gesellschaftspolitisch „konservative“ Politikvorstellungen repräsentieren.

Bezüglich des Einflusses gegenläufiger Parteiorientierungen der Stadtratskoalition und des Oberbürgermeisters wird eine Dummy-Variable „Kohabitation“erstellt, die den Wert 1 annimmt, wenn die potenzielle Koalition nicht die Partei des Oberbürgermeisters enthält. Mit Daten zur Zusammensetzung der nordrhein-westfälischen

13 Allerdings sind bei keiner Partei große programmatische „Sprünge“ von einer Kommunalwahl zur nächsten zu beobachten. So verändert zwar beispielsweise die FDP in Mönchengladbach ihre policy-Positionen zwischen 1999 und 2009; dies geschieht jedoch in überschaubarem Maße. Wirtschaftspolitische Positionen der FDP Mönchengladbach: 17,7 (1999), 18,8 (2004), 18,1 (2009); gesellschaftspolitische Positionen: 5,6 (1999), 6,7 (1999), 7,1 (2009). 
Landesregierungen und durch die Berechnung der Differenz zwischen dem Sitzanteil jeder Partei (Wählervereinigung bzw. Einzelbewerber) im Landtag und im Stadtrat kann bestimmt werden, ob sich die erwarteten Effekte hinsichtlich der Kongruenz kommunaler und regionaler Koalitionen zeigen (siehe Ştefuriuc 2013).

Zur Identifizierung der Faktoren, die einen Einfluss auf die Koalitionsbildung haben, verwende ich konditionale Logit-Modelle, die auch auf lokaler Ebene bereits zur Analyse von Koalitionsbildungen verwendet wurden (Bäck 2003; Skjæveland/ Serritzlew/Blom-Hansen 2007; Martin/Stevenson 2001, 2010; McFadden 1974). ${ }^{14}$ Jeder Koalitionsbildungsprozess nach einer Wahl wird durch ein sogenanntes choice set dargestellt. Dieses beinhaltet auf Basis der Sitzverteilung im Stadtrat nicht nur die tatsächlich gebildete Koalition (die den Wert „1“ auf der abhängigen Variablen erhält), sondern auch alle anderen Kombinationen von Koalitionen (die den Wert „0“ auf der abhängigen Variablen erhalten). Die Anzahl aller möglichen Koalitionskombinationen ist damit durch die Anzahl der im Stadtrat vertretenen Akteure bedingt. Deshalb variiert die Größe des choice set über die Fälle hinweg.

\section{Empirische Analysen}

In diesem Abschnitt wird zunächst ein Überblick über die Anzahl an Koalitionsbildungen sowie die Koalitionskonstellationen in 29 nordrhein-westfälischen Großstädten mit mindestens 100.000 Einwohnern zwischen 1999 und 2009 gegeben. Dabei gilt es zuerst die Frage zu beantworten, inwieweit die kommunalpolitischen Akteure die Koalitionsmuster der übergeordneten politischen Ebenen „kopieren“. Anschließend wird überprüft, welche Faktoren zur Erklärung kommunaler Koalitionsbildungen beitragen.

Zwischen 1999 und 2009 wurden in den nordrhein-westfälischen Großstädten insgesamt 55 Koalitionen gebildet (vgl. Tabelle 1). Die große Mehrheit der Koalitionen sind dabei minimal winning coalitions. Minderheitskoalitionen und „übergroße" Koalitionen werden nur selten gebildet. Bei 48 von 55 Bündnissen war dabei die Partei des Oberbürgermeisters Mitglied der Koalition. Somit gibt es nur sieben

14 Die konditionalen Logit-Modelle passen bei der Analyse kommunaler Koalitionsbildungsprozesse in nordrhein-westfälischen Großstädten besser zur Datenstruktur als die von Glasgow, Golder und Golder (2012) präsentierten Mixed Logit-Modelle zur Analyse parlamentarischer Regierungsbildungen. Dies ist durch die Fokussierung auf die kommunale Koalitionsbildung in einem einzigen Bundesland bedingt, denn es bestehen a priori keine theoretischen Erwartungen hinsichtlich des Auftretens unbeobachteter Heterogenität in den geschätzten Effekten zwischen den einzelnen nordrhein-westfälischen Großstädten, da das institutionelle Setting (Kommunalverfassung, Kommunalwahlrecht) konstant ist (vgl. ähnlich Klingelhöfer/Müller 2013). Bei der Analyse der konditionalen Logit-Modelle wurde dem Vorgehen von Martin und Stevenson (2001) gefolgt. 
„Kohabitationssituationen“, in denen die Partei des Oberbürgermeisters nicht Teil der Stadtratskoalition war. ${ }^{15}$ Die deskriptiven Daten unterstützen damit die Hypothese, dass die kommunalpolitischen Akteure bei der Koalitionsbildung einen Anreiz haben, die Partei des Oberbürgermeisters in die Koalition miteinzubeziehen.

Tabelle 1: Koalitionsbildung in 29 nordrhein-westfälischen Großstädten, 1999-2009

\begin{tabular}{lcrr|c}
\hline & 1999 & 2004 & 2009 & Total \\
\hline Anzahl Koalitionen & 13 & 22 & 20 & 55 \\
\hline Kleinstmögliche Gewinnkoalitionen & 10 & 16 & 17 & 43 \\
Minderheitskoalitionen & 2 & 5 & 3 & 10 \\
Übergroße Koalitionen & 1 & 1 & 0 & 2 \\
\hline Partei Oberbürgermeister Koalitionsmitglied & 10 & 20 & 18 & 48 \\
Kongruenz zur Landesregierung & 3 & 9 & 8 & 20 \\
\hline
\end{tabular}

Hinsichtlich der Hypothesen zur Kongruenz kommunaler Koalitionen zu den jeweiligen Landesregierungen zeigen die deskriptiven Daten kein einheitliches Bild. Während die kommunalpolitischen Akteure in nahezu drei Viertel aller Fälle kleinstmögliche Gewinnkoalitionen eingehen, entsprechen die gebildeten Koalitionen nur selten dem zum gleichen Zeitpunkt existierenden Koalitionsmuster auf der Landesebene. Kommunale Koalitionen in nordrhein-westfälischen Großstädten sind in vielen Fällen inkongruent zu den jeweiligen Landesregierungen, die kommunalpolitischen Akteure „kopieren“ jedoch das gängige Koalitionsformat auf regionaler (und nationaler) Ebene, nämlich vor allem schwarz-gelbe und rot-grüne minimal winning coalitions (vgl. Abbildung 3).

Die durchschnittliche Anzahl an Parteien, die an einer Koalition beteiligt sind, liegt bei 2,2 (vgl. Tabelle 2). Dies deutet bereits darauf hin, dass die kommunalpolitischen Akteure aus Gründen des office-seeking nicht nur minimal winning coalitions präferieren, sondern kleinstmögliche Gewinnkoalitionen mit der geringsten Anzahl an Koalitionspartnern. Die deskriptiven Informationen zeigen weiterhin, dass infolge der Abschaffung der Fünfprozenthürde 1999 sowohl die durchschnittliche Anzahl kommunalpolitischer Akteure als auch der Fragmentierungsgrad im Stadtrat deutlich ansteigen (vgl. auch Gehne 2013) und die kommunalen Parteien-

152009 gab es keine Stichwahlen zur Bestimmung der Oberbürgermeister, sodass die Oberbürgermeister- und Stadtratswahlen hier immer gekoppelt waren (abgesehen von Düsseldorf). Die geäußerte Vermutung, dass Kohabitationen vor allem dann zustande kommen, wenn die Oberbürgermeister erst in der Stichwahl gewählt werden (Holtkamp 2008: 250), die Stadtrats- und Oberbürgermeisterwahlen demnach entkoppelt sind (bspw. 1999 und 2004), wird durch die Daten unterstützt: Vier von fünf „Kohabitationssituationen“ finden sich bei entkoppelten Wahlen. 
Abbildung 3: Koalitionen in nordrhein-westfälischen Großstädten, 1999-2009

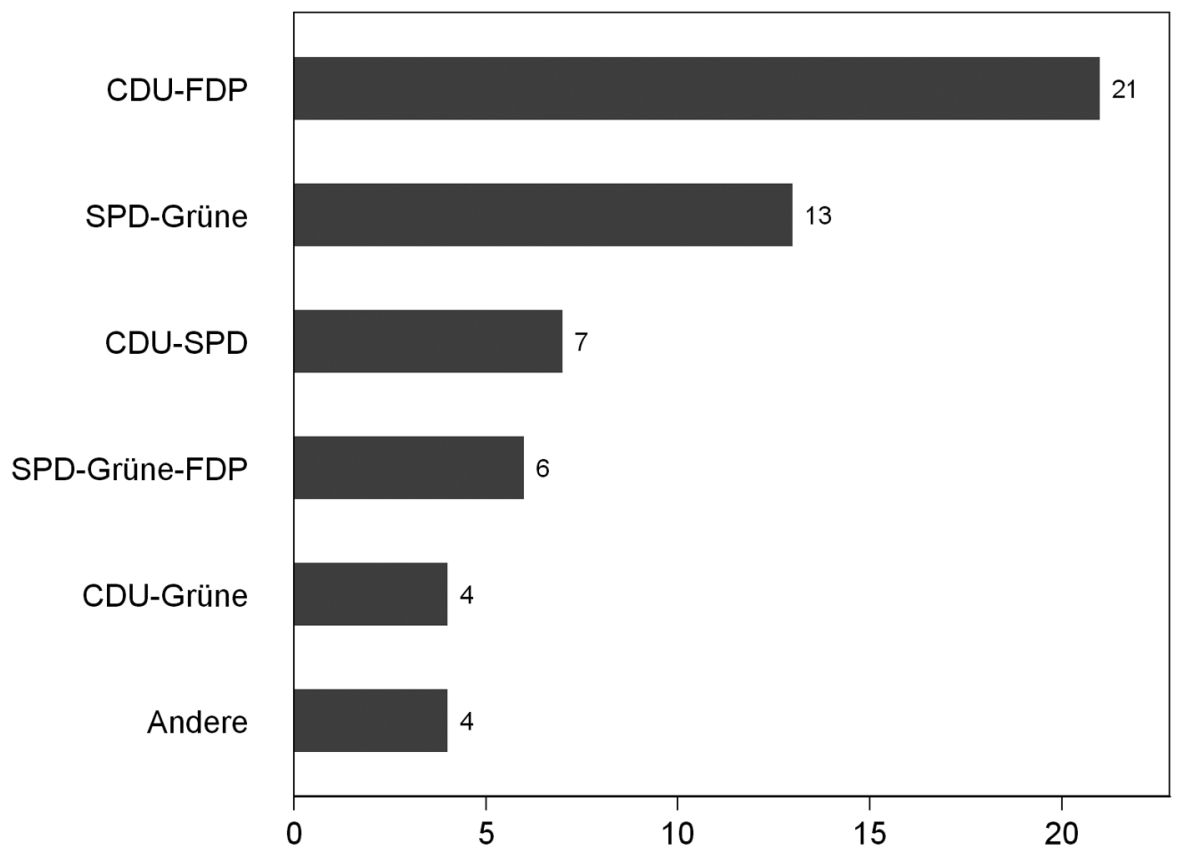

systeme dem Landesparteiensystem zunehmend unähnlicher werden. ${ }^{16}$ Der erhöhte Fragmentierungsgrad erschwert jedoch die Koalitionsbildung nicht in dem Sinne, dass weniger Koalitionen gebildet werden - im Gegenteil: Die kommunalpolitischen Akteure gehen immer häufiger schriftlich fixierte Bündnisse ein (vgl. Tabelle 1). Zum einen dürfte dies daran liegen, dass nach dem Wegfall der Fünfprozenthürde absolute Mehrheiten im Stadtrat für eine Partei noch unwahrscheinlicher geworden sind. Zum anderen kann bei durchschnittlich sieben bis acht politischen Gruppierungen im Stadtrat nur noch selten mit wechselnden Mehrheiten gearbeitet werden, da die kommunalpolitischen Akteure mit steigender Anzahl an Akteuren immer unsicherer hinsichtlich der Strategien anderer Akteure werden. Folglich streben sie danach, diese „Unsicherheit“" durch Koalitionen auf Basis schriftlicher Vereinba-

16 Der Fragmentierungsgrad im Stadtrat wurde anhand der „effektiven Parteienzahl“ von Golosov (2010) mithilfe der Sitzanteile der kommunalpolitischen Gruppierungen im Stadtrat berechnet. Gegenüber der bisher gebräuchlichen Berechnung der „effektiven Parteienzahl“ nach Laakso und Taagepera (1979) zeichnet sich Golosovs Index durch methodische Vorteile und eine „intuitivere“ Interpretation aus. 
rungen zu verringern. Dies geschieht zunehmend in kleinstmöglichen Gewinnkoalitionen, die aus drei Parteien bestehen. Die damit ansteigende Anzahl an Vetospielern innerhalb der Koalition (Holtkamp 2008: 276) hält die kommunalpolitischen Akteure dabei nicht von der Koalitionsbildung ab.

Tabelle 2: Deskriptive Informationen zu den Koalitionen in nordrhein-westfälischen Großstädten, 1999-2009

\begin{tabular}{lcccc}
\hline & 1999 & 2004 & 2009 & $1999-2009$ \\
\hline $\begin{array}{l}\text { Durchschnittliche Asymmetrie der } \\
\text { kommunalen Parteiensysteme (im Stadtrat) }\end{array}$ & 0,32 & 0,35 & 0,45 & 0,38 \\
$\begin{array}{l}\text { Durchschnittliche Anzahl kommunalpolitischer } \\
\text { Akteure im Stadtrat }\end{array}$ & 5,54 & 7,55 & 7,50 & 7,05 \\
$\begin{array}{l}\text { Durchschnittlicher Fragmentierungsgrad im Stadtrat } \\
\text { Durchschnittliche Anzahl Koalitionspartner }\end{array}$ & 2,43 & 3,18 & 3,46 & 3,10 \\
\hline
\end{tabular}

Welche Faktoren sind nun für die kommunale Koalitionsbildung in nordrhein-westfälischen Großstädten entscheidend? Tabelle 3 zeigt die Ergebnisse von vier konditionalen Logit-Modellen, die sich hinsichtlich der Einbeziehung der unabhängigen Variablen voneinander unterscheiden. Modell 1 umfasst die „klassischen“ Theorien der Koalitionsbildung, das heißt die office- und policy-seeking-Variablen. Die Ergebnisse entsprechen größtenteils den theoretischen Erwartungen. Der politische Prozess in nordrhein-westfälischen Großstädten ist hauptsächlich von der Ämterorientierung der Akteure geprägt. Potenzielle Koalitionen sind signifikant wahrscheinlicher, wenn es sich um minimal winning coalitions handelt. Darüber hinaus bevorzugen die kommunalpolitischen Akteure solche Koalitionen, die so wenige Parteien wie möglich zur Erreichung einer Stadtratsmehrheit umfassen.

Die Variablen zur Veto-Spieler-Distanz zwischen den ideologisch „extremsten“ Parteien innerhalb potenzieller Koalitionen weisen das erwartete negative Vorzeichen auf. Allerdings erreicht nur die Variable zur gesellschaftspolitischen Politikdistanz ein gebräuchliches statistisches Signifikanzniveau. Dieser Effekt ist über die Modelle hinweg stabil. Potenzielle Koalitionen sind demnach weniger wahrscheinlich, wenn die Koalitionspartner deutlich unterschiedliche policy-Vorstellungen hinsichtlich gesellschaftspolitischer Fragen haben. 


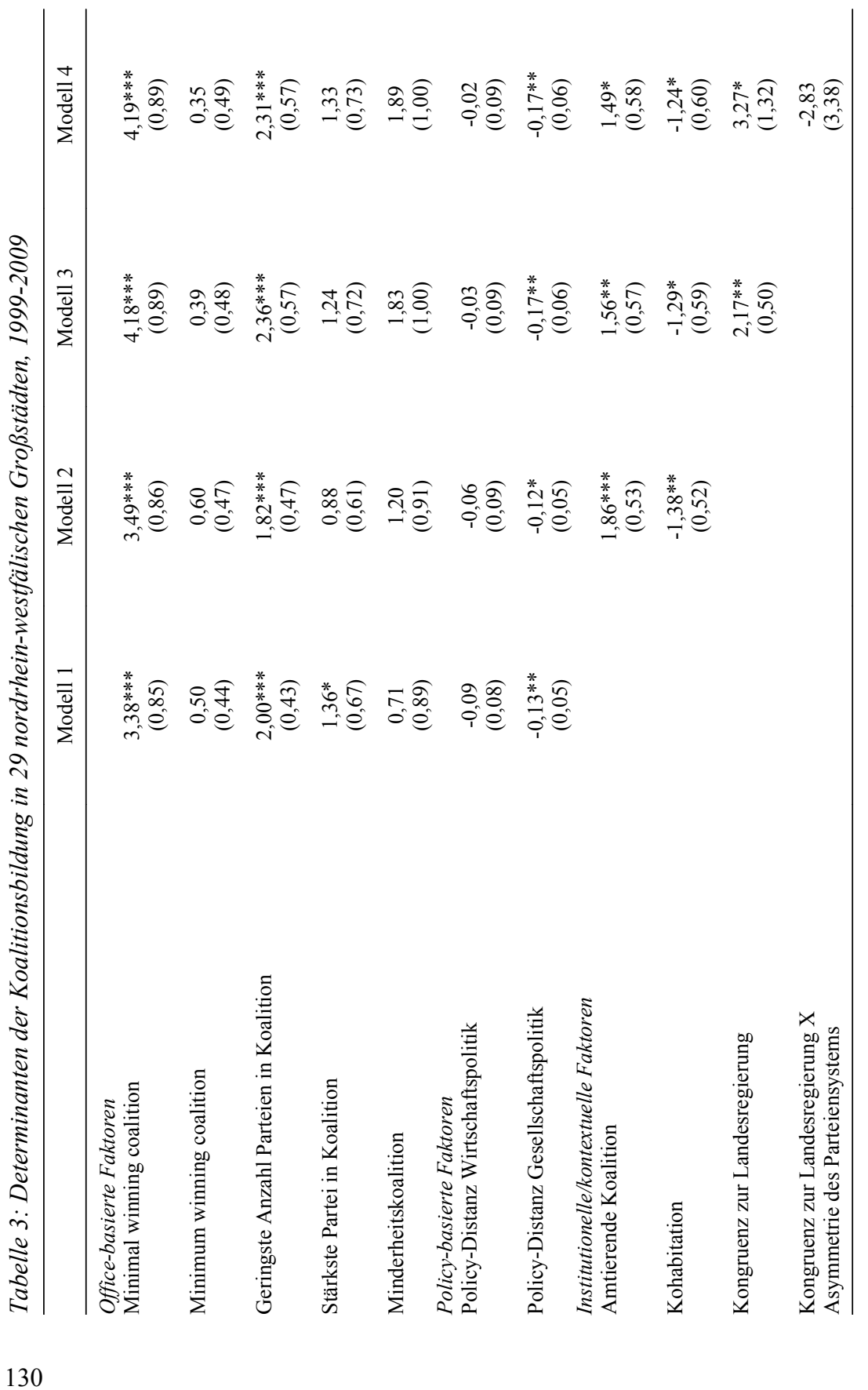




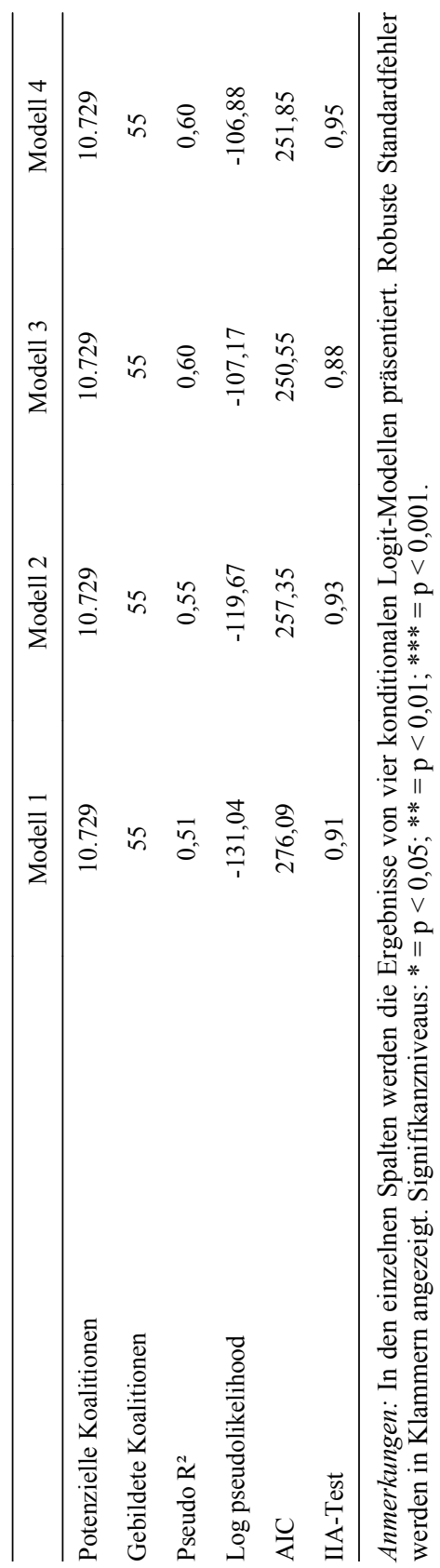


Der Einbezug der stärksten Partei in potenzielle Koalitionen erhöht scheinbar signifikant die Wahrscheinlichkeit, dass diese Koalitionen gebildet werden. Die entsprechende Variable hat in Modell 1 einen positiven und statistisch signifikanten Koeffizienten. Der Blick auf die Modelle 2-4 zeigt jedoch, dass dieser Effekt nur wirksam wird, wenn nicht für die Partei des Oberbürgermeisters kontrolliert wird. Es ist vor allem die Partei des Oberbürgermeisters, die in Koalitionen einbezogen wird, und nicht die stärkste Partei, auch wenn beides oftmals miteinander einhergeht. Die Hypothese hinsichtlich des Anreizes für die kommunalpolitischen Akteure, die Partei des Oberbürgermeisters in die Koalition miteinzubeziehen, um „Kohabitationssituationen“ zu vermeiden, wird damit bestätigt: Die Variable hat einen statistisch signifikanten und negativen Effekt auf die Koalitionsbildungswahrscheinlichkeit. Potenzielle Koalitionen sind wahrscheinlicher, wenn sie die Partei des Oberbürgermeisters einschließen.

Lokale institutionelle Faktoren (Modell 2) haben ebenso wie kontextuelle Faktoren, die sich aus dem deutschen Mehrebenensystem ergeben (Modelle 3 und 4), eine große Bedeutung bei der kommunalen Koalitionsbildung. Wie auch in anderen Studien zur lokalen Koalitionsbildung (Bäck 2003; Skjæveland/Serritzlew/BlomHansen 2007) sind potenzielle Koalitionen wahrscheinlicher, wenn die Koalitionspartner bereits in der vorherigen Legislaturperiode miteinander zusammengearbeitet haben. Darüber hinaus sind potenzielle Koalitionen wahrscheinlicher, wenn sie sich aus den Parteien der Landesregierung zusammensetzen (Modell 3). Die kommunalpolitischen Akteure streben folglich danach, zur Landesregierung kongruente Stadtratskoalitionen zu bilden. Sie tun dies allerdings unabhängig vom Asymmetriegrad des kommunalen Parteiensystems (Modell 4). ${ }^{17}$

Zusammenfassend lässt sich festhalten, dass die Koalitionsbildungen in nordrhein-westfälischen Großstädten sowohl hinsichtlich einzelner Koalitionskonstellationen als auch mit Blick auf die Determinanten der Koalitionsbildung ähnlichen Mustern folgen wie auf der Bundes- und Landesebene. Zwar spielen auch lokale institutionelle constraints wie der direkt gewählte Oberbürgermeister und Mehrebenenfaktoren eine wichtige Rolle; nichtsdestoweniger streben die kommunalpolitischen Akteure vor allem danach, Ämter zu kontrollieren und ihre programmatisch-

17 In einem Regressionsmodell müssten alle Bestandteile eines Interaktionsterms zusätzlich zur Interaktionsvariablen in ein Modell aufgenommen werden (vgl. Brambor/Clark/Golder 2006). In konditionalen Logit-Modellen ist dies jedoch nicht möglich, wenn Bestandteile eines Interaktionsterms nicht über die verschiedenen Alternativen variieren, da diese Variablen ansonsten aus der Analyse entfernt werden würden. In Modell 4 können deshalb nicht alle Bestandteile des Interaktionsterms aufgenommen werden, da die jeweilige Asymmetrie des kommunalen Parteiensystems nicht über die Koalitionsalternativen hinweg variiert, sondern innerhalb einer Koalitionsbildungssituation für alle Koalitionsalternativen konstant ist. 
ideologischen Vorstellungen bestmöglich durchzusetzen. Dabei werden überwiegend Bündnisse zwischen den ,üblichen Verdächtigen“ gebildet.

\section{Fazit}

Ziel des vorliegenden Beitrags war eine deskriptive und empirische Analyse kommunaler Koalitionsbildung und ihrer Determinanten in 29 nordrhein-westfälischen Großstädten zwischen 1999 und 2009. Es wurde argumentiert, dass auch die kommunalpolitischen Akteure an prestigeträchtigen Ämtern und an der Umsetzung ihrer programmatisch-ideologischen Vorstellungen interessiert sind, sowie dass sie das Ziel haben, ihren Wählerstimmenanteil zu maximieren. Darüber hinaus wurde angenommen, dass institutionelle und kontextuelle Faktoren die kommunale Koalitionsbildung beeinflussen könnten.

Die empirischen Ergebnisse bestätigen weitgehend die Erwartungen. Die Bildung kommunaler Koalitionen weist große Ähnlichkeiten mit der Koalitionsbildung auf Bundes- und Landesebene auf. Sowohl office- als auch policy-orientierte Faktoren spielen neben institutionellen Faktoren bei der Koalitionsbildung eine Rolle. Auch in den Stadträten werden überwiegend die bekannten Zweiparteienkoalitionen aus SPD-Bündnis 90/Die Grünen und CDU-FDP gebildet. Kleinstmögliche Gewinnkoalitionen, die die geringste mögliche Anzahl an Parteien umfassen, werden eher gebildet als Minderheits- oder ,übergroße“ Koalitionen. Darüber hinaus sind Koalitionen umso wahrscheinlicher, je geringer die policy-Distanz zwischen den Koalitionspartnern auf der gesellschaftspolitischen Dimension ist. Des Weiteren werden oftmals die bereits amtierenden Bündnisse nach den Kommunalwahlen weitergeführt. Zusätzlich lässt sich zeigen, dass die kommunalpolitischen Akteure bei der Bildung von Stadtratskoalitionen die Rolle des Oberbürgermeisters als weiteren, entscheidenden Akteur im Koalitionsbildungsprozess berücksichtigen, denn kommunale Koalitionen sind weniger wahrscheinlich, wenn es zu „Kohabitationssituationen" kommen kann, das heißt wenn die Partei des Oberbürgermeisters nicht Teil der Stadtratskoalition ist. Kommunale Koalitionen sind zudem wahrscheinlicher, wenn sie kongruent zur Landesregierung sind.

Inwieweit lassen sich diese Ergebnisse über die nordrhein-westfälischen Großstädte hinaus verallgemeinern? Für einen ersten Test der Koalitionstheorien auf kommunaler Ebene in Deutschland wurde sich zunächst auf ein Kommunalsystem konzentriert, dessen institutionelle Rahmenbedingungen im Vergleich zu Bayern oder Baden-Württemberg mit der Einführung von Oberbürgermeisterdirektwahlen in den 1990er Jahren vor nicht allzu langer Zeit geändert wurde, und wo es somit keine jahrzehntelang eingespielten Abläufe zwischen Stadtratsmehrheit und direkt 
gewähltem Oberbürgermeister geben kann. Gegenläufige Parteiorientierungen zwischen Ratskoalition und (Ober-)Bürgermeister existieren jedoch nicht nur in nordrhein-westfälischen Großstädten. 2001 war dies in knapp einem Drittel aller deutschen Städte und Gemeinden der Fall (Fischer 2002: 18). Weitere Untersuchungen könnten anhand der Analyse ,typischer“ und „abweichender“ Fälle (Bäck/Dumont 2007; Lieberman 2005) die kausalen Prozesse der (Nicht-)Einbeziehung der Partei des Oberbürgermeisters in Stadtratskoalitionen verdeutlichen.

Dass office- und policy-seeking-Gründe auch in Großstädten anderer Kommunalsysteme eine Rolle spielen sollten, zeigt das Beispiel der Koalitionsbildung im Münchner Stadtrat 2014. Vor den Kommunalwahlen warb der bayerische Ministerpräsident Horst Seehofer (CSU) offen für eine schwarz-grüne Koalition im Münchner Stadtrat. ${ }^{18}$ „Schwarz-Grün“ erreichte jedoch keine eigene Mehrheit. Eine zunächst angestrebte Koalition aus Bündnis 90/Die Grünen, CSU und SPD scheiterte anschließend vor allem an der Weigerung der Bündnisgrünen, der CSU das Vorschlagsrecht für die Besetzung des Amts des Kreisverwaltungsreferenten zu überlassen. ${ }^{19}$ Schlussendlich bildete sich eine minimal winning coalition aus CSU und SPD. Auch in weiteren bayerischen Großstädten bildeten sich nach den Kommunalwahlen 2014 Koalitionen im Stadtrat (bspw. Augsburg, Nürnberg). Dies lässt sich auch in den hessischen Großstädten in den letzten zwanzig Jahren beobachten (prominenteste Beispiele sind die schwarz-grüne Koalition in Frankfurt am Main sowie das grün-schwarze Bündnis in Darmstadt).

Hinsichtlich der Gemeindegröße lassen sich die empirischen Ergebnisse sicherlich nicht auf kleinste Gemeinden verallgemeinern, in denen nur wenige politische Akteure im Gemeinderat sitzen und sich damit allein zahlenmäßig eine feste Kooperation einzelner Akteure nicht rechnet. Die mit steigender Gemeindegröße zunehmende Parteipolitisierung macht es hingegen wahrscheinlicher, dass sich die gezeigten Erklärungsfaktoren kommunaler Koalitionsbildung auch in mittleren und größeren Städten mit weniger als 100.000 Einwohnern finden lassen. So wurden in so unterschiedlichen Gemeinden und Städten wie dem hessischen Bensheim 2011 (knapp 40.000 Einwohner), dem rheinland-pfälzischen Limburgerhof 2009 (knapp 11.000 Einwohner), dem bayerischen Königsbrunn 2014 (knapp 27.000 Einwohner) oder auch dem sächsischen Görlitz 2009 (knapp 54.000 Einwohner) in verschiedenen Kommunalsystemen schriftliche Kooperationsvereinbarungen hinsichtlich gemeinsamer Ratsbündnisse unterzeichnet, die von ,Schwarz-Grün“(Bensheim) über 
große Koalitionen (Limburgerhof) bis zu „Jamaika“-Koalitionen (Görlitz, Königsbrunn) reichen.

Die Koalitionsbildungsprozesse in deutschen Kommunen sollten demnach auch in anderen Bundesländern mit unterschiedlichen Kommunalsystemen und weniger kompetitivem Parteienwettbewerb (Egner/Krapp/Heinelt 2013; Holtkamp 2008) getestet werden, da sich ,weiterhin sehr gravierende Unterschiede zwischen den Kommunalverfassungen konstatieren" lassen (Bogumil/Holtkamp 2006: 61). Zum einen könnte der Einfluss unterschiedlicher institutioneller Rahmenbedingungen auf die kommunale Koalitionsbildung vergleichend in den Blick genommen werden. Zum anderen bieten sich auch vergleichende Analysen hinsichtlich des Einflusses der Gemeindegröße auf die einzelnen Determinanten kommunaler Koalitionsbildung an. Darüber hinaus existiert eine Forschungslücke bei der Frage, in welchem Ausmaß in kleineren Gemeinden Koalitionen im Gemeinderat gebildet werden und welche Faktoren bei diesen Koalitionsbildungen ausschlaggebend sind. Die theoretischen Erwartungen und empirischen Ergebnisse des vorliegenden Beitrags können als Ausgangspunkt für diese weitergehenden Untersuchungen dienen.

\section{Literatur}

Austen-Smith, David/Banks, Jeffrey, 1988: Elections, Coalitions and Legislative Outcomes, in: American Political Science Review 82 (2), 405-422.

Axelrod, Robert, 1970: Conflict of Interest. A Theory of Divergent Goals with Applications to Politics, Chicago.

Bäck, Hanna, 2003: Explaining and predicting coalition outcomes: Conclusions from studying data on local coalitions, in: European Journal of Political Research 42 (4), 441-472.

Bäck, Hanna, 2008: Intra-Party Politics and Coalition Formation: Evidence from Swedish Local Government, in: Party Politics 14 (1), 71-89.

Bäck, Hanna, 2009: Intra-party politics and local coalition formation, in: Daniela Giannetti/Kenneth Benoit (Hrsg.), Intra-Party Politics and Coalition Governments in Parliamentary Democracies, London/New York, 53-68.

Bäck, Hanna/Debus, Marc/Müller, Jochen/Bäck, Henry, 2013: Regional Government Formation in Varying Multilevel Contexts: A Comparison of Eight European Countries, in: Regional Studies 47 (3), 368-387.

Bäck, Hanna/Dumont, Patrick, 2007: Combining Large-n and Small-n strategies: The Way Forward in Coalition Research, in: West European Politics 30 (3), 467-501. 
Bäck, Hanna/Dumont, Patrick, 2008: Making the first move. A two-stage analysis of the role of formateurs in parliamentary government formation, in: Public Choice 135 (3), 353-373.

Bäck, Henry, 2005: The institutional setting of local political leadership and community involvement, in: Michael Haus/Hubert Heinelt/Murray Stewart (Hrsg.), Urban Governance and Democracy, London/New York, 65-101.

Baron, David P./Diermeier, Daniel, 2001: Elections, Governments, and Parliaments in Proportional Representation Systems, in: Quarterly Journal of Economics 116 (3), 933-967.

Baron, David P./Ferejohn, John A., 1989: Bargaining in Legislatures, in: American Political Science Review 83 (4), 1181-1206.

Benoit, Kenneth/Laver, Michael, 2006: Party Policy in Modern Democracies, London.

Bogumil Jörg, 2001: Modernisierung lokaler Politik. Kommunale Entscheidungsprozesse im Spannungsfeld zwischen Parteienwettbewerb, Verhandlungszwängen und Ökonomisierung, Baden-Baden.

Bogumil, Jörg, 2010: Parteien in der Kommunalpolitik. Hoffnungsträger oder Auslaufmodell?, in: David H. Gehne/Tim Spier (Hrsg.), Krise oder Wandel der Parteiendemokratie?, Wiesbaden, 37-48.

Bogumil, Jörg/Grohs, Stephan/Holtkamp, Lars, 2010: Zersplitterte Kommunalparlamente oder Stärkung lokaler Demokratie? Warum die Abschaffung der kommunalen Fünfprozenthürde in Nordrhein-Westfalen ein Fehler war, in: Zeitschrift für Parlamentsfragen 41 (4), 788-802.

Bogumil, Jörg/Holtkamp, Lars, 2006: Kommunalpolitik und Kommunalverwaltung. Eine policyorientierte Einführung, Wiesbaden.

Bogumil, Jörg/Holtkamp, Lars, 2013: Kommunalpolitik und Kommunalverwaltung. Eine praxisorientierte Einführung, Bonn.

Brambor, Thomas/Clark, William Roberts/Golder, Matt, 2006: Understanding Interaction Models: Improving Empirical Analyses, in: Political Analysis 14 (1), 63-82.

Bräuninger, Thomas/Debus, Marc, 2012: Parteienwettbewerb in den deutschen Bundesländern, Wiesbaden.

Brearey, Patricia, 1989: City Coalitions in West Germany: A Case-Study of Nordrhein-Westfalen, in: Colin Mellors/Bert Pijnenburg (Hrsg.), Political parties and coalitions in European local government, London, 275-300.

Buzogány, Aron/Kropp, Sabine, 2013: Koalitionen von Parteien, in: Oskar Niedermayer (Hrsg.), Handbuch Parteienforschung, Wiesbaden, 261-293. 
Koalitionsbildung in deutschen Großstädten

Camões, Pedro J./Mendes, Silvia M., 2009: Party politics and local government coalition formation in Portugal, in: Daniela Giannetti/Kenneth Benoit (Hrsg.), Intra-Party Politics and Coalition Governments in Parliamentary Democracies, London/New York, 69-85.

Carey, John, 2007: Competing Principals, Political Institutions, and Party Unity in Legislative Voting, in: American Journal of Political Science 51 (1), 92-107.

Carey, John, 2009: Legislative Voting and Accountability, Cambridge.

Däubler, Thomas/Debus, Marc, 2009: Government Formation and Policy Formulation in the German States, in: Regional and Federal Studies 19 (1), 73-95.

De Swaan, Abram, 1973: Coalition theories and cabinet formations. A study of formal theories of coalition formation applied to nine European parliaments after 1918, Amsterdam.

Debus, Marc/Knill, Christoph/Tosun, Jale, 2012: Drum zahle, wer sich ewig bindet: Eine Analyse der Gebührenhöhe für eingetragene Lebenspartnerschaften in Baden-Württemberg, in: Politische Vierteljahresschrift 53 (1), 1-28.

Debus, Marc/Knill, Christoph/Tosun, Jale, 2013 a: Registration fees for Same-Sex Unions, Local Party Politics and Societal Demand, in: Local Government Studies 39 (6), 756-776.

Debus, Marc/Knill, Christoph/Tosun, Jale, 2013 b: Verwaltungsgebühren als Instrument symbolischer Steuerung: Kirchenaustrittsgebühren in baden-württembergischen Kommunen, in: dms - der moderne Staat 6 (1), 191-209.

Denters, Bas, 1985: Towards a Conditional Model of Coalition Behaviour, in: European Journal of Political Research 13 (3), 295-309.

Deschouwer, Kris, 2003: Political Parties in Multi-Layered Systems, in: European Urban and Regional Studies 10 (3), 213-226.

Deschouwer, Kris, 2006: Political Parties as Multi-Level Organizations, in: Richard S. Katz/William J. Crotty (Hrsg.), Handbook of Party Politics, London, 291-300. Downs, William M., 1998: Coalition Government, Subnational Style. Multiparty Politics in Europe's Regional Parliaments, Columbus.

Egner, Björn/Krapp, Max-Christopher/Heinelt, Hubert, 2013: Das deutsche Gemeinderatsmitglied. Problemsichten - Einstellungen - Rollenverständnis, Wiesbaden.

Fischer, Josef, 2002: Bürgermeister und Stadträte in Deutschland 2001. CDU/CSU sind die kommunale Kraft, in: Stadtforschung und Statistik 2, 15-18.

Florack, Martin, 2010: Politische Willensbildung mit wechselnden Mehrheiten?, in: Forum Kommunalpolitik 1, 12-14. 
Franklin, Mark N./Mackie, Thomas T., 1983: Familiarity and Inertia in the Formation of Governing Coalitions in Parliamentary Democracies, in: British Journal of Political Science 13 (3), 275-298.

Freitag, Markus/Vatter, Adrian, 2010: Vergleichende subnationale Analysen für Deutschland. Institutionen, Staatstätigkeit und politische Kulturen, Münster.

Frey, Rainer/Naßmacher, Karl-Heinz, 1975: Parlamentarisierung der Kommunalpolitik?, in: Archiv für Kommunalwissenschaften 14 (2), 192-212.

Fried, Robert C., 1976: Party and Policy in West German Cities, in: American Political Science Review 70 (1), 11-24.

Gabriel, Oscar W., 1984: Parlamentarisierung der Kommunalpolitik, in: Oscar W. Gabriel/Peter Haungs/Matthias Zender (Hrsg.), Opposition in Großstadtparlamenten, Melle, 101-147.

Gabriel, Oscar W., 2000: Introduction, in: Oscar W. Gabriel/Vincent HoffmannMartinot/Hank V. Savitch (Hrsg.), Urban Democracy, Opladen, 9-27.

Gehne, David H., 2013: Ent-Partei-Politisierung der lokalen repräsentativen Demokratie? Eine Analyse am Beispiel der Ratswahlen in NRW 1979 bis 2009, in: Michael Haus/Sabine Kuhlmann (Hrsg.), Lokale Politik und Verwaltung im Zeichen der Krise?, Wiesbaden, 49-63.

Gehne, David H./Holtkamp, Lars, 2005: Fraktionsvorsitzende und Bürgermeister in NRW und Baden-Württemberg, in: Jörg Bogumil/Hubert Heinelt (Hrsg.), Bürgermeister in Deutschland, Wiesbaden, 87-141.

Geys, Benny/Heyndels, Bruno/Vermeir, Jan, 2006: Explaining the formation of minimal coalitions: Anti-system parties and anti-pact rules, in: European Journal of Political Research 45 (6), 957-984.

Giannetti, Daniela/Benoit, Kenneth, 2009: Intra-party politics and coalition governments in parliamentary democracies, in: Daniela Giannetti/Kenneth Benoit (Hrsg.), Intra-Party Politics and Coalition Governments in Parliamentary Democracies, London/New York, 3-24.

Glasgow, Garrett, 2001: Mixed Logit Models for Multiparty Elections, in: Political Analysis 9 (1), 116-136.

Glasgow, Garrett/Golder, Matt/Golder, Sona N., 2012: New Empirical Strategies for the Study of Parliamentary Government Formation, in: Political Analysis 20 (2), 248-270.

Goerres, Achim/Tepe, Markus, 2013: Für die Kleinen ist uns nichts zu teuer? Kindergartengebühren und ihre Determinanten in Deutschlands 95 bevölkerungsreichsten Städten zwischen 2007 und 2010, in: dms - der moderne Staat 6 (1), 169-190. 
Golosov, Grigorii V., 2010: The Effective Number of Parties. A New Approach, in: Party Politics 16 (2), 171-192.

Gravdahl, Hans Petter, 1998: Consensual Coalitions? Coalition Formation in Norwegian Municipalities, in: Scandinavian Political Studies 21 (4), 307-323.

Grofman, Bernard, 1982: A Dynamic Model of Protocoalition Formation in Ideological N-Space, in: Behavioral Science 27 (1), 77-90.

Hogwood, Patricia, 1999: Playing to win. Adapting Concepts of Rationality and Utility for the German Coalition Context, in: Roland Sturm/Sabine Kropp (Hrsg.), Hinter den Kulissen von Regierungsbündnissen, Baden-Baden, 15-43. Holtkamp, Lars, 2008: Kommunale Konkordanz- und Konkurrenzdemokratie. Parteien und Bürgermeister in der repräsentativen Demokratie, Wiesbaden.

Holtmann, Everhard, 1993: Parteien in der lokalen Politik, in: Roland Roth/Hellmut Wollmann (Hrsg.), Kommunalpolitik, Bonn, 256-270.

Holtmann, Everhard, 2002: Parteien und Wählergruppen in der Kommunalpolitik, in: Oscar W. Gabriel/Oskar Niedermayer/Richard Stöss (Hrsg.), Parteiendemokratie in Deutschland, Wiesbaden (2., aktualisierte und erweiterte Aufl.), 406-427.

Holtmann, Everhard, 2004: Haben politische Parteien in der Kommunalpolitik noch eine Zukunft? Zur Vereinbarkeit parteienstaatlicher Konkurrenzdemokratie und kommunaler Selbstverwaltung, in: Werner K. Blessing/Heinrich Pehle (Hrsg.), Die Zukunftsfähigkeit der Stadt in Vergangenheit und Gegenwart, Erlangen, 225-245.

Jeffery, Charlie, 2008: The challenge of territorial politics, in: Policy \& Politics 36 (4), 545-557.

Kang, Shin-Goo, 2009: The influence of presidential heads of state on government formation in European democracies: Empirical evidence, in: European Journal of Political Research 48 (4), 543-572.

Killguss, Hans-Peter/Peters, Jürgen/Häusler, Alexander, 2008: PRO Köln - Entstehung und Aktivitäten, in: Alexander Häusler (Hrsg.), Rechtspopulismus als „Bürgerbewegung“, Wiesbaden, 55-71.

Kleinert, Hubert, 2004: Schwarz-Grün erweitert Optionen. Zur Auflösung ideologischer Tabus, in: Die Politische Meinung 413, 69-74.

Klingelhöfer, Tristan/Müller, Jochen, 2013: Consociational and rational coalitions: Norm-based government formation and the case of the Dutch provinces, in: Acta Politica (doi:10.1057/ap.2013.36), 13.12.2013.

Knemeyer, Franz-Ludwig, 1999: Gemeindeverfassungen, in: Hellmut Wollmann/ Roland Roth (Hrsg.), Kommunalpolitik, Opladen, 104-122. 
Kuschke, Wolfram/Cryns, Manfred, 1984: Kommunalwahlen in Nordrhein-Westfalen, in: Uwe Andersen (Hrsg.), Kommunalpolitik und Kommunalwahlen in Nordrhein-Westfalen, Düsseldorf, 59-94.

Laakso, Markku/Taagepera, Rein, 1979: „Effective“ Number of Parties: A Measure with Application to West Europe, in: Comparative Political Studies 12 (1), 3-27.

Laver, Michael, 1989: Theories of Coalition Formation and Local Government Coalitions, in: Colin Mellors/Bert Pijnenburg (Hrsg.), Political parties and coalitions in European local government, London, 15-33.

Laver, Michael/Benoit, Kenneth/Garry, John, 2003: Extracting Policy Positions from Political Texts Using Words as Data, in: American Political Science Review 92 (2), 311-331.

Laver, Michael/Rallings, Colin/Thrasher, Michael, 1987: Coalition Theory and Local Government: Coalition Payoffs in Britain, in: British Journal of Political Science 17 (4), 501-509.

Laver, Michael/Rallings, Colin/Thrasher, Michael, 1998: Policy Payoffs in Local Government, in: British Journal of Political Science 28 (2), 333-353.

Laver, Michael/Schofield, Norman, 1998: Multiparty Government. The Politics of Coalition in Europe, Oxford.

Leiserson, Michael Avery, 1968: Factions and Coalitions in One-Party Japan - An Interpretation Based on the Theory of Games, in: American Political Science Review 62 (3), 770-787.

Lieberman, Evan S., 2005: Nested Analysis as a Mixed-Method Strategy for Comparative Research, in: American Political Science Review 99 (3), 435-452.

Lowe, Will, 2008: Understanding Wordscores, in: Political Analysis 16 (4), 356-371. Lupia, Arthur/Strøm, Kaare, 2010: Bargaining, Transaction Costs, and Coalition Governance, in: Kaare Strøm/Wolfgang C. Müller/Torbjörn Bergman (Hrsg.), Cabinets and Coalition Bargaining, Oxford, 51-83.

Martin, Lanny W./Stevenson, Randolph T., 2001: Government Formation in Parliamentary Democracies, in: American Journal of Political Science 45 (1), 33-50. Martin, Lanny W./Stevenson, Randolph T., 2010: The Conditional Impact of Incumbency on Government Formation, in: American Political Science Review 104 (3), 503-518.

Martinussen, Pål E., 2002: In Search of the Government in Local Government: Coalition Agreements and Office Payoffs in Norway, in: Scandinavian Political Studies 25 (2), 139-171.

McFadden, Daniel, 1974: Conditional Logit Analysis of Qualitative Choice Behavior, in: Paul Zarembka (Hrsg.), Frontiers in Econometrics, New York, 105-142. 
Mellors, Colin/Brearey, Patricia, 1986: Multi-dimensional approaches to the study of local coalitions: some cross-national comparisons, in: Geoffrey Pridham (Hrsg.), Coalitional behaviour in theory and practice, Cambridge, 278-295.

Müller, Jochen, 2013: On a Short Leash? Sub-National Party Positions between Regional Context and National Party Unity, in: Journal of Elections, Public Opinion and Parties 23 (2), 177-199.

Müller, Wolfgang C., 2000: Political Parties in Parliamentary Democracies: Making Delegation and Accountability Work, in: European Journal of Political Research 37 (3), 309-333.

Neu, Alexander S., 2004: Schwarz-grüner „Tabubruch“. Zur prinzipiellen Koalitionsoffenheit auf allen politischen Ebenen, in: Die Politische Meinung 419, 61-68.

Nienaber, Georg, 2004: Direkt gewählte Bürgermeister in Nordrhein-Westfalen. Positionierung zwischen Bürgern, Politik und Verwaltung im Fokus von Effektivierung und Demokratisierung der lokalen Ebene, Marburg.

Ott, Yvonne, 1994: Der Parlamentscharakter der Gemeindevertretung. Eine rechtsvergleichende Untersuchung der Qualität staatlicher und gemeindlicher Vertretungskörperschaften, Baden-Baden.

Pappi, Franz Urban, 2009: Regierungsbildung im deutschen Fünf-Parteiensystem, in: Politische Vierteljahresschrift 50 (2), 187-202.

Pappi, Franz Urban/Shikano, Susumu, 2004: Ideologische Signale in den Wahlprogrammen der deutschen Bundestagsparteien 1980 bis 2002, MZES Working Papers 76.

Peters, Jürgen/Sager, Thomas/Häusler, Alexander, 2008: PRO NRW und PRO D - Entwicklung, Struktur und Methodik, in: Alexander Häusler (Hrsg.), Rechtspopulismus als „Bürgerbewegung“, Wiesbaden, 72-87.

Revelt, David/Train, Kenneth, 1998: Mixed Logit with Repeated Choices: Household's Choices of Appliance Efficiency Level, in: Review of Economics and Statistics 80 (4), 647-657.

Riker, William H., 1962: The Theory of Political Coalitions, New Haven/London. Schmidt-Eichstaedt, Gerd, 1985: Die Machtverteilung zwischen der Gemeindevertretung und dem Hauptverwaltungsbeamten im Vergleich der deutschen Kommunalverfassungssysteme, in: Archiv für Kommunalwissenschaften 24 (1), 20-36.

Schniewind, Aline, 2010: Kommunale Parteiensysteme zwischen Mehrheits- und Verhandlungsdemokratie. Ein Vergleich der Kreise und kreisfreien Städte Deutschlands, in: Markus Freitag/Adrian Vatter (Hrsg.), Vergleichende subna- 
tionale Analysen für Deutschland. Institutionen, Staatstätigkeit und politische Kulturen, Münster, 131-176.

Serritzlew, Søren/Blom-Hansen, Jens/Skjoeveland, Asbjørn, 2010: Portfolio Allocation or Policy Horizons? Determinants of Coalition Formation in Danish Local Governments, in: Local Government Studies 36 (6), 843-866.

Serritzlew, Søren/Skjaeveland, Asbjørn/Blom-Hansen, Jens, 2008: Explaining Oversized Coalitions: Empirical Evidence from Local Governments, in: The Journal of Legislative Studies 14 (4), 421-450.

Skjceveland, Asbjørn/Serritzlew, Søren/Blom-Hansen, Jens, 2007: Theories of coalition formation: An empirical test using data from Denmark, in: European Journal of Political Research 46 (5), 721-745.

Snyder, Richard, 2001: Scaling Down. The Subnational Comparative Method, in: Studies in Comparative International Development 36 (1), 93-110.

Ştefuriuc, Irina, 2009 a: Government Formation in Multi-Level Settings: Spanish

Regional Coalitions and the Quest for Vertical Congruence, in: Party Politics 15 (1), 93-115.

Ştefuriuc, Irina, 2009 b: Governing Strategies in Multilevel Settings: Coordination, Innovation or Territorialization?, in: Wilfried Swenden/Bart Maddens (Hrsg.), Territorial Party Politics in Western Europe, London, 183-203.

Ştefuriuc, Irina, 2013: Government Formation in Multi-Level Settings. Party Strategy and Institutional Constraints, Houndmills, Basingstoke.

Steunenberg, Bernard, 1992: Coalition theories: Empirical evidence for Dutch municipalities, in: European Journal of Political Research 22 (3), 245-278.

Stoiber, Michael/Egner, Björn, 2008: Ein übertragbarer Amtsinhaber-Bonus bei Kommunalwahlen. Eine vergleichende Analyse in drei Bundesländern, in: Zeitschrift für Vergleichende Politikwissenschaft 2 (2), 287-314.

Strøm, Kaare/Budge, Ian/Laver, Michael, 1994: Constraints on Cabinet Formation in Parliamentary Democracies, in: American Journal of Political Science 38 (2), 303-335.

Strøm, Kaare/Nyblade, Benjamin, 2007: Coalition theory and government formation, in: Carles Boix/Susan C. Stokes (Hrsg.), The Oxford handbook of comparative politics, Oxford, 782-802.

Swenden, Wilfried/Maddens, Bart (Hrsg.), 2009: Territorial Party Politics in Western Europe, London.

Tausendpfund, Markus, 2013: Gemeinden als Rettungsanker der EU? Individuelle und kontextuelle Faktoren der Zustimmung der Bürgerinnen und Bürger zur Europäischen Union, Baden-Baden.

Train, Kenneth, 2003: Discrete Choice Methods with Simulation, Cambridge. 
Tsebelis, George, 1995: Decision Making in Political Systems: Veto Players in Presidentialism, Parliamentarism, Multicameralism and Multipartyism, in: British Journal of Political Science 25 (3), 289-325.

Tsebelis, George, 2002: Veto Players: How Political Institutions Work, Princeton. van Deth, Jan W./Tausendpfund, Markus (Hrsg.), 2013: Politik im Kontext: Ist alle

Politik lokale Politik? Individuelle und kontextuelle Determinanten politischer Orientierungen, Wiesbaden.

van Houten, Pieter, 2009: Multi-Level Relations in Political Parties. A Delegation Approach, in: Party Politics 15 (2), 137-156.

Vetter, Angelika/Kuhn, Sebastian, 2013: (Nationale) Parteien in der lokalen Politik:

Wandel oder Krise?, in: Michael Haus/Sabine Kuhlmann (Hrsg.), Lokale Politik und Verwaltung im Zeichen der Krise?, Wiesbaden, 27-48.

von Neumann, John/Morgenstern, Oskar, 1944: Theory of games and economic behaviour, Princeton.

Wehling, Hans-Georg, 1991: „Parteipolitisierung“ von lokaler Politik und Verwaltung? Zur Rolle der Parteien in der Kommunalpolitik, in: Hubert Heinelt/Hellmut Wollmann (Hrsg.), Brennpunkt Stadt, Basel, 149-166.

Wehling, Hans-Georg, 2010: Rat und Bürgermeister in der deutschen Kommunalpolitik. Ein Rückblick auf die Reformprozesse, in: Andreas Kost/Hans-Georg Wehling (Hrsg.), Kommunalpolitik in den deutschen Ländern, Wiesbaden (2., aktualisierte und überarbeitete Aufl.), 353-366.

Zeuner, Bodo/Wischermann, Jörg, 1995: Rot-Grün in den Kommunen. Konfliktpotentiale und Reformperspektiven: Ergebnisse einer Befragung von Kommunalpolitikern, Opladen.

Korrespondenzanschrift:

Martin Gross, M.A.

Professur Politische Wissenschaft III

Universität Mannheim

A 5, 6

68131 Mannheim

E-Mail: martin.gross@uni-mannheim.de 